\title{
QUEEN'S
UNIVERSITY
BELFAST
}

\section{The effect of community-based interventions for cardiovascular disease secondary prevention on behavioural risk factors}

Lawlor, E. R., Bradley, D. T., Cupples, M. E., \& Tully, M. A. (2018). The effect of community-based interventions for cardiovascular disease secondary prevention on behavioural risk factors. Preventive Medicine, 114, 24-38. https://doi.org/10.1016/j.ypmed.2018.05.019

Published in:

Preventive Medicine

Document Version:

Peer reviewed version

Queen's University Belfast - Research Portal:

Link to publication record in Queen's University Belfast Research Portal

Publisher rights

Copyright 2018 Elsevier

This manuscript is distributed under a Creative Commons Attribution-NonCommercial-NoDerivs License

(https://creativecommons.org/licenses/by-nc-nd/4.0/), which permits distribution and reproduction for non-commercial purposes, provided the author and source are cited.

\section{General rights}

Copyright for the publications made accessible via the Queen's University Belfast Research Portal is retained by the author(s) and / or other copyright owners and it is a condition of accessing these publications that users recognise and abide by the legal requirements associated with these rights.

Take down policy

The Research Portal is Queen's institutional repository that provides access to Queen's research output. Every effort has been made to ensure that content in the Research Portal does not infringe any person's rights, or applicable UK laws. If you discover content in the Research Portal that you believe breaches copyright or violates any law, please contact openaccess@qub.ac.uk. 
1 The effect of community-based interventions for cardiovascular disease

2 secondary prevention on behavioural risk factors.

3 Dr Emma R Lawlor, ${ }^{*}$ UKCRC Centre of Excellence for Public Health (Northern

4 Ireland), School of Medicine, Dentistry and Biomedical Sciences, Queen's University

5 Belfast, Clinical Sciences Block B, Royal Victoria Hospital, Belfast, Northern Ireland,

6 UK.

$7 \quad$ Email: elawlor01@qub.ac.uk

8 Dr. Declan T Bradley, UKCRC Centre of Excellence for Public Health (Northern

9 Ireland), School of Medicine, Dentistry and Biomedical Sciences, Queen's University

10 Belfast, Northern Ireland, UK.

11 Public Health Agency Northern Ireland, Belfast, Northern Ireland, UK.

12 Email: dbradley09@qub.ac.uk

13 Professor Margaret E Cupples, UKCRC Centre of Excellence for Public Health

14 (Northern Ireland), School of Medicine, Dentistry and Biomedical Sciences, Queen's

15 University Belfast, Belfast, Northern Ireland, UK.

16 Department of General Practice and Primary Care, Queen's University Belfast,

17 Belfast, Northern Ireland, UK.

18 Email: m.cupples@qub.ac.uk

19 Dr. Mark A Tully, UKCRC Centre of Excellence for Public Health (Northern Ireland),

School of Medicine, Dentistry and Biomedical Sciences, Queen's University Belfast, 
21 Belfast, Northern Ireland, UK.

22 Email: m.tully@qub.ac.uk

23 Corresponding author: Dr Emma Lawlor, elawlor01@qub.ac.uk

24 Word Count: 3254

* Present address: MRC Epidemiology Unit, University of Cambridge School of Clinical Medicine, Box 285 Institute of Metabolic Science, Cambridge Biomedical

27 Campus, Cambridge, CB2 0QQ, UK.

\section{ABSTRACT}

Cardiovascular disease (CVD) is the leading cause of death worldwide, and its prevalence is increasing; with limited healthcare resources, secondary prevention programmes outside traditional hospital settings are needed, but their effectiveness is unclear. We aimed to assess the effectiveness of secondary prevention cardiovascular risk reduction programmes delivered in venues situated within the community on modification of behavioural risk factors. We searched five databases (MEDLINE, EMBASE, CINAHL, PsycINFO, Cochrane library) to identify trials of health behaviour interventions for adults with CVD in community-based venues. Primary outcomes were changes in physical activity, diet, smoking and/or alcohol consumption. Two reviewers independently assessed articles for eligibility and risk of bias; statistical analysis used Revman v5.3. Of 5905 articles identified, 41 articles (38 studies) ( $n=7970)$ were included. Interventions were mainly multifactorial, educational, psychological and physical activity-based. Meta-analyses identified increased steps/week (Mean Difference (MD): 7480; 95\% CI 1940, 13020) and minutes of physical activity/week (MD: 59.96; 95\% CI 15.67, 104.25) associated with interventions. There was some evidence for beneficial effects on peak $\mathrm{VO}_{2}$, blood 
pressure, total cholesterol and mental health. Variation in outcome measurements reported for other behavioural risk factors limited our ability to perform metaanalyses. Effective interventions were based in homes, general practices or outpatient settings, individually tailored and often multicomponent with a theoretical framework. Our review identified evidence that interventions for secondary CVD prevention, delivered in various community-based venues, have positive effects on physical activity; such opportunities should be promoted by health professionals.

\section{Highlights}

- Our meta-analysis provides strong evidence that interventions for secondary CVD prevention, delivered in community-based venues, are effective in promoting PA; evidence for beneficial effects on peak VO2, blood pressure, total cholesterol and mental health is less clear.

- Evidence of their effectiveness on other behavioural risk factors is limited due to heterogeneity of reported outcome measurements.

- Effective interventions were individually tailored, based in homes, general practices or outpatient settings and tended to be multicomponent with a theoretical framework.

\section{INTRODUCTION}

Globally, cardiovascular disease (CVD) is the leading cause of mortality.[1] CVD morbidity rates are also rapidly rising, with an estimated worldwide prevalence of 200.5 million in 2015.[2] This has had large direct and indirect social and economic consequences, costing the UK economy in 2015 approximately £24.0 billion.[3] Although secondary prevention and cardiac rehabilitation (CR) can reduce CVD morbidity and mortality, their uptake is poor; in the UK, only $47 \%$ of patients attend 
CR after a cardiac event.[4] Reasons for lack of participation include travel distance, belief in ability to manage their condition alone and lack of time.[5] Many individuals with CVD fail to change their behavioural risk factors and there is a need for improved methods of delivering secondary prevention services.[6] The use of nontraditional healthcare settings (such as community centres, churches and leisure centres) and home-based programmes in helping to overcome barriers and improve uptake of secondary CVD prevention has been studied. Clark et al.'s (2010)[7] review of 39 randomised control trials (RCTs) on home-based secondary prevention programmes for coronary heart disease $(\mathrm{CHD})$ found small to moderate significant improvements for quality of life, systolic blood pressure, smoking cessation, total cholesterol and depression. Devi et al. (2015)[8] review of RCTs evaluating internet delivered secondary interventions for $\mathrm{CHD}$ found some evidence for beneficial effects on quality of life, dietary outcomes and PA. However, both reviews found studies were of low quality and there was much heterogeneity in outcome measures used. Furthermore, previous systematic reviews have focused on particular settings (e.g. participants' homes),[7] specific behavioural risk factors (e.g. smoking)[9] or different modes of delivery (e.g. internet).[8,10,11] There is a lack of evidence for the relative effectiveness of interventions which involve various modes of delivery in different venues situated within the community, on multiple behavioural risk factors. Thus, we aimed to conduct a systematic review, including meta-analysis to examine the effectiveness of interventions, delivered in community-based venues, on modification of behavioural risk factors in the secondary prevention of CVD. We also included biophysical outcomes, mental and physical health measures and total mortality in our analyses in order to gain insight into the potential wider health benefits of the included studies. 
METHODS

95

96

97

98

99

100

101

102

103

104

105

106

107

108

109

110

111

112

113

114

115

116

\section{Protocol \& registration}

We designed the review protocol (www.crd.york.ac.uk/PROSPERO; registration no. CRD42015030014) based on the PRISMA statement[12]

\section{Eligibility criteria}

We considered studies to be eligible if participants were community-dwelling adults aged $\geq 18$ years with a CVD diagnosis. Interventions needed to have a lifestyle/behaviour change focus for secondary CVD prevention and address one or more of: physical activity (PA), diet, smoking and/or alcohol. Comparisons were either no intervention or minimal intervention. Eligible studies were those that had interventions delivered within a venue situated in the community, including general practices, participants' homes and community centres, excluding hospitals.

Community and population level interventions were not eligible for inclusion. Primary outcomes were the change of a behavioural risk factor for CVD: PA, diet, smoking and/or alcohol consumption. Secondary outcomes included peak $\mathrm{VO}_{2}$, blood pressure, total cholesterol, body mass index (BMI), waist circumference, mental and physical health, and total mortality. We included studies with a minimum of three months' follow-up from baseline; study designs included randomised controlled trials (RCT), cluster RCTs, quasi-experimental designs using a control population for comparison, interrupted time-series studies, and prospective controlled cohort studies.[13] Limits were set to publications in English language but no regional restrictions were applied.

\section{Information sources}


117 We conducted searches in MEDLINE, EMBASE, CINAHL, PsycINFO and Cochrane

118 library from January 2005 to 8 th June, 2015 related to the concepts: CVD, healthrelated behaviours, preventive interventions[14] and study design[13] (Appendix 1).

120 Relevant terms were searched as subject headings, and key words relating to the 121 subject headings were entered as truncated terms (using *), and/or searched for as adjacent terms (using "adj") (title and abstract). Terms relating to the concept of setting were not included in the search to avoid potentially excluding or misclassifying settings, especially in different countries. We searched reference lists of relevant systematic reviews for other potentially eligible studies.

\section{Study selection}

127

We imported results from searches into Refworks (v3.1, ProQuest, U.S.A.) and removed duplicates. Study titles and abstracts were screened independently by $E R L$ and DTB. We obtained full text papers that were deemed potentially relevant and also screened independently for eligibility. In cases of disagreement or uncertainty, we reached consensus via a third reviewer (MEC or MAT).

\section{Data collection process}

Data were extracted from our included studies independently by ERL and DTB and cross-checked for consistency. If studies provided data for multiple follow-up time points, we extracted data for the time furthest from baseline. We made attempts to contact authors to retrieve missing data.

\section{Risk of bias}

ERL and DTB assessed the studies independently, using the Cochrane Collaboration's tool for assessing risk of bias,[15] as being 'high', 'low' or 'unclear' for each criterion and overall. Due to the nature of the studies, blinding of participants 
was not always feasible therefore we assessed 'Blinding of participants, personnel and outcome assessors' rather than blinding of participants alone.

\section{Synthesis of results}

144 We analysed data using Review Manager (RevMan version 5.3; Nordic Cochrane 145 Centre, Copenhagen, Denmark). We used the random effects model to acknowledge heterogeneity; unstandardized mean differences were used in analysis and $95 \%$ confidence intervals $(\mathrm{Cl})$ were reported. We tested statistical heterogeneity using the $I^{2}$ statistic and categorised heterogeneity into: low (0\% to $\left.30 \%\right)$, moderate $(30 \%$ to $60 \%$ ), substantial (60\% to $90 \%$ ) and considerable (90\% to $100 \%)$. We categorised follow-up from baseline outcome assessment times into subgroups of: 3 to 6 months, 7 to 12 months and >12 months.

\section{Additional analysis}

Five studies presented their outcome data as mean change from baseline; all other studies reported the follow-up measurement values. To include data from these five studies in our analyses, we added/subtracted, as appropriate, values for change to/from the baseline means and used the standard deviation (SD) for the baseline mean in initial meta-analyses. Sensitivity analyses were conducted excluding these studies. We also conducted further sensitivity analyses, in which we excluded studies deemed to be at high risk of bias overall.

\section{RESULTS}

Our electronic database searching yielded 5905 papers; three were added from reference lists of systematic reviews (Figure 1). We removed duplicates, leaving 5758 papers for title and abstract screening; full text versions of 157 papers were assessed. In total, 41 articles, reporting 38 studies, met our inclusion criteria. Six 
articles[16-21] reported the outcomes of three studies; for each study, the earlier article was used as the study reference. Common reasons for exclusion were participants' age (<18 years), no reported control group, no outcomes relevant to this review and lack of behaviour change intervention.

\section{Study characteristics}

Studies included 7970 participants with a mean age of 62.3 years (SD 5.3) and $78 \%$ of participants were male. Participants' diagnoses were reported as coronary heart disease (CHD),[22-32] acute coronary syndrome (ACS)[19,33-38] and myocardial infarction (MI).[16,39-46] Only one study was specifically aimed at socioeconomically deprived communities.[18]

The majority of interventions were multicomponent, with PA, psychological and educational content (Table 1). For fourteen studies, the main focus was on increasing PA,[29,31,32,36,38,39,44,46-52] one focused on smoking cessation[28] and one on uptake of Mediterranean diet.[42] All others ( $n=22)$ focused on a combination of behavioural risk factors. Only fifteen studies reported using a theoretical framework, including the Social Cognitive $(n=7)[16,26,32,36,44,50,51]$ and Transtheoretical Models $(n=3)$. $[23,44,53]$ Most studies recruited participants from hospitals, as inpatients or outpatients, and six recruited from general practice.[17,22,26,47,48,54]

The majority of interventions $(n=25)$ were home-based: three of these used exclusively internet delivery, $[18,29,54] 14$ used exclusively telephone delivery, $[16,23,24,28,31,38,41,43,44,50-52,55,56]$ two used both online and telephone delivery,[34,48] two used printed information[32,46] and four used homevisits[39,42,45,53] with one of these also including telephone contact.[39] Seven 
studies were general practice/primary care-based.[19,22,26,33,37,40,47] Various professional disciplines were involved in intervention delivery, most frequently nurses $(n=15),[22,26,28,30,35-37,41,43,45,47,52-54,56]$ doctors $(n=10),[19,26,30,33,34,37,39,41,46,47]$ dieticians $(n=4)[23,35,40,42]$ and physiotherapists $(n=3) \cdot[29,39,49]$ Studies varied in length of follow-up: most were less than 12 months after baseline.

Twenty-seven studies used a two-group RCT (four of these were described as prospective),([16,37,41,43]) seven a three-group RCT design,[24,32,40,42,52,55,56] two a cluster RCT,[26,48] one was a pilot study[28] and one was a follow-up of a

198 RCT by review of national datasets.[22] In regards to the 3-group RCT studies, one study[24] had two intervention groups that could be combined into a single group following Cochrane collaboration guidance.[57] Two studies[25,28] had two eligible

201 intervention groups but had outcomes that could not be pooled. The remaining 202 studies[18,29,34,42] had one intervention group that was eligible for inclusion. 
Table 1; Characteristics of included studies

\begin{tabular}{|c|c|c|c|c|c|c|c|c|c|}
\hline $\begin{array}{l}\text { Study/ } \\
\text { Country }\end{array}$ & \begin{tabular}{|c|} 
Study \\
population + \\
source
\end{tabular} & $\begin{array}{c}\text { Mean age } \\
\left(\text { years) }^{*}\right.\end{array}$ & $\begin{array}{l}\text { Sample } \\
\text { size }\end{array}$ & $\begin{array}{c}\text { Study } \\
\text { design }\end{array}$ & $\begin{array}{l}\text { Intervention (Duration, content, } \\
\text { intensity, theoretical framework, } \\
\text { professional involvement) }\end{array}$ & Control & Outcomes & Follow-up & $\begin{array}{c}\text { Mode of } \\
\text { delivery of } \\
\text { intervention }\end{array}$ \\
\hline \multicolumn{10}{|c|}{ Setting: Participants' homes } \\
\hline \multicolumn{10}{|c|}{ Internet based delivery } \\
\hline $\begin{array}{l}\text { Devi et al. } \\
\text { (2014),[54] } \\
\text { England }\end{array}$ & $\begin{array}{l}\text { Patients with } \\
\text { stable angina; } \\
\text { GP CHD } \\
\text { register, } \\
\text { postal } \\
\text { invitation }\end{array}$ & $\begin{array}{l}\text { I: } 66 \text { (SD 8) } \\
\text { C: } 66 \text { (SD } \\
10)\end{array}$ & $\begin{array}{l}\mathrm{I}: 36 \\
\mathrm{C}: 37\end{array}$ & $\begin{array}{l}\text { Randomized } \\
\text { Controlled } \\
\text { Trial (RCT) }\end{array}$ & $\begin{array}{l}6 \text { wks: Web-based programme } \\
\text { (introductory home visit); tailored goals for } \\
\text { PA, diet, managing emotions \& smoking } \\
\text { review via website, email or chatroom } \\
\text { contact with cardiac nurses. }\end{array}$ & $\begin{array}{l}\text { Usual GP care } \\
\text { (CHD annual } \\
\text { check) }\end{array}$ & $\begin{array}{l}\text { Primary: Step count } \\
\text { Secondary: sedentary \& } \\
\text { moderate PA, EE, } \\
\text { weight, SBP, DBP, body } \\
\text { fat, diet, anxiety, } \\
\text { depression, SE (general; } \\
\text { diet; knowledge); SEE } \\
\text { disease perception, } \\
\text { satisfaction, HRQoL } \\
\end{array}$ & $\begin{array}{l}6 \text { wks \& } 6 \\
\text { mths }\end{array}$ & $\begin{array}{l}\text { Interactive } \\
\text { website, email } \\
\& \text { chatroom }\end{array}$ \\
\hline $\begin{array}{l}\text { Lindsay et } \\
\text { al. (2008; } \\
2009),[18 \\
17] \\
\text { England }\end{array}$ & $\begin{array}{l}\text { Patients on } \\
\text { GP CHD } \\
\text { register }\end{array}$ & Overall: 63 & $\begin{array}{l}\text { I: } 54 \\
\text { C: } 54\end{array}$ & $\mathrm{RCT}$ & $\begin{array}{l}9 \text { mths: Given computer, broadband } \\
\text { subscription, access to health portal; wkly } \\
\text { drop-in sessions; phone-in support. For } 6 \\
\text { mths:,moderator-led discussion forums \& } \\
\text { one-to-one instant messaging }\end{array}$ & $\begin{array}{l}\text { Computer; } \\
\text { broadband } \\
\text { subscription, } \\
\text { wkly drop-in } \\
\text { sessions; } \\
\text { phone-in } \\
\text { support }\end{array}$ & $\begin{array}{l}\text { Moderate PA, alcohol, } \\
\text { smoking, second-hand } \\
\text { smoke, diet, healthcare } \\
\text { visits, sources of info, } \\
\text { social support, mental } \\
\text { health, confidence in } \\
\text { managing health, health } \\
\text { locus of control }\end{array}$ & $\begin{array}{l}6 \mathrm{mths} \\
\& 9 \mathrm{mths}\end{array}$ & $\begin{array}{l}\text { Website } \\
\text { health portal }\end{array}$ \\
\hline $\begin{array}{l}\text { Reid et al. } \\
\text { (2012),[29] } \\
\text { Canada }\end{array}$ & $\begin{array}{l}\text { Patients with } \\
\text { CHD; not } \\
\text { accessing } \\
\text { CR; hospital }\end{array}$ & $\begin{array}{l}\text { Overall: } 56 \\
\text { (SD 9) } \\
\text { I: } 57(9) \\
\text { C: } 56(9)\end{array}$ & $\begin{array}{l}\mathrm{I}: 115 \\
\mathrm{C}: 108\end{array}$ & $\mathrm{RCT}$ & $\begin{array}{l}6 \text { mths: Tailored PA plan; website access } \\
\text { for PA tracking; } 5 \text { online tutorials (wks } 2,4 \text {, } \\
\text { 8, } 14 \text { \& 20); emails with exercise specialist. }\end{array}$ & $\begin{array}{l}\text { Usual care, } \\
\text { cardiologist: } \\
\text { PA guidance \& } \\
\text { education } \\
\text { booklet }\end{array}$ & $\begin{array}{l}\text { Primary: Step count } \\
\text { Secondary: } \leq \text { moderate } \\
\text { leisure time PA, HRQoL, } \\
\text { smoking, medication }\end{array}$ & $6 \& 12 \mathrm{mths}$ & $\begin{array}{l}\text { Interactive } \\
\text { website \& } \\
\text { email }\end{array}$ \\
\hline \multicolumn{10}{|c|}{ Telephone based delivery } \\
\hline $\begin{array}{l}\text { Butler et } \\
\text { al. } \\
(2009),[50] \\
\text { Australia }\end{array}$ & $\begin{array}{l}\text { Patients at } \\
\text { CR OP } \\
\text { programme }\end{array}$ & $\begin{array}{l}\text { I: } 63(\text { SD 10) } \\
\text { C: } 65(11)\end{array}$ & $\begin{array}{l}\text { I: } 44 \\
\text { C: } 46\end{array}$ & $\mathrm{RCT}$ & $\begin{array}{l}6 \text { wks: PA self-monitoring (pedometer); } \\
\text { behavioural counselling \& goal setting via } \\
\text { telephone; } 2 \text { PA info brochures; based on } \\
\text { SCT. }\end{array}$ & $\begin{array}{l}2 \text { PA info } \\
\text { brochures }\end{array}$ & $\begin{array}{l}\text { Primary: Total PA \& } \\
\text { walking sessions } \\
\text { Secondary: SEE, } \\
\text { outcome expectancies, } \\
\text { self-management, } \\
\text { psychological distress, } \\
\text { METS weight, WC. }{ }^{\star *} \\
\end{array}$ & $\begin{array}{l}6 \text { wks \& } 6 \\
\text { mths }\end{array}$ & $\begin{array}{l}\text { Telephone } \\
\text { calls }\end{array}$ \\
\hline Furber et & Cardiac & I: 67 (SD 11) & I: 95 & $\mathrm{RCT}$ & See Butler et al. (2009) & See Butler et & Primary: Total PA \& & See Butler et & See Butler et \\
\hline
\end{tabular}




\begin{tabular}{|c|c|c|c|c|c|c|c|c|c|}
\hline $\begin{array}{l}\text { al. } \\
\text { (2010),[51] } \\
\text { Australia }\end{array}$ & $\begin{array}{l}\text { patients; } \\
\text { from referral } \\
\text { database }\end{array}$ & C: 65 (12) & C: 106 & & & al. (2009) & $\begin{array}{l}\text { walking sessions } \\
\text { Secondary: SEE, } \\
\text { outcome expectancies, } \\
\text { self-management, } \\
\text { psychological distress }\end{array}$ & al. (2009) & al. (2009) \\
\hline $\begin{array}{l}\text { Hanssen } \\
\text { et al. } \\
\text { (2007),[41] } \\
\text { Norway }\end{array}$ & $\begin{array}{l}\text { Patients with } \\
\text { acute Ml; } \\
\text { hospital }\end{array}$ & $\begin{array}{l}\text { I: } 61 \text { (SD 11) } \\
\text { C: } 60 \text { (13) }\end{array}$ & $\begin{array}{l}\text { I: } 97 \\
\text { C: } 96\end{array}$ & $\begin{array}{l}\text { Prospective } \\
\text { RCT }\end{array}$ & $\begin{array}{l}6 \text { mths: Nurse-led telephone (wks } 1-4,6,8 \text {, } \\
12 \& 24) \& \text { open telephone line: info on risk } \\
\text { reduction, emotional support, coping, goal } \\
\text { setting, GP/cardiologist review; theoretical } \\
\text { basis (Lazarus \& Folkman). }\end{array}$ & $\begin{array}{l}\text { Usual care } \\
\text { (physician OP } \\
\text { clinic at 6-8 } \\
\text { wks; GP) }\end{array}$ & $\begin{array}{l}\text { Primary: HRQoL } \\
\text { Secondary: Smoking, PA } \\
\text { sessions }\end{array}$ & $3 \& 6$ mths & $\begin{array}{l}\text { Telephone } \\
\text { calls }\end{array}$ \\
\hline $\begin{array}{l}\text { Hawkes et } \\
\text { al. } \\
(2013) ;[16] \\
\text { Turkstra et } \\
\text { al. } \\
\text { (2013),[21] } \\
\text { Australia } \\
\end{array}$ & $\begin{array}{l}\text { Patients with } \\
\text { MI or PCl; } \\
\text { hospital }\end{array}$ & $\begin{array}{l}\text { I: } 61 \text { (SD 11) } \\
\text { C: } 60 \text { (11) }\end{array}$ & $\begin{array}{l}\text { I: } 156 \\
\text { C: } 170\end{array}$ & $\begin{array}{l}\text { Parallel } \\
\text { group } \\
\text { prospective } \\
\text { RCT }\end{array}$ & $\begin{array}{l}6 \text { mths: Up to } 10 \times 30 \mathrm{~min} \text { telephone health } \\
\text { coaching sessions: CHD risk factor profile, } \\
\text { feedback on risk profile, SMART goals; } \\
\text { follow-up with usual healthcare providers; } \\
\text { posted educational resources; based on } \\
\text { SCT. }\end{array}$ & $\begin{array}{l}\text { Usual care, } \\
\text { 'My Heart My } \\
\text { Life' } \\
\text { educational } \\
\text { resource; } \\
\text { \& quarterly } \\
\text { newsletter }\end{array}$ & $\begin{array}{l}\text { Primary: HRQoL, total PA } \\
\text { Other: Diet, BMI, } \\
\text { smoking, QALY, } \\
\text { healthcare visits, } \\
\text { satisfaction, hospital } \\
\text { admissions, medication }\end{array}$ & $6 \mathrm{mths}$ & $\begin{array}{l}\text { Telephone } \\
\text { calls, posted } \\
\text { printed info \& } \\
\text { healthcare } \\
\text { providers if } \\
\text { required }\end{array}$ \\
\hline $\begin{array}{l}\text { Lear et al. } \\
\text { (2006),[23] } \\
\text { Canada }\end{array}$ & $\begin{array}{l}\text { Patients with } \\
\text { ischemic } \\
\text { heart } \\
\text { disease; } \\
\text { hospital }\end{array}$ & $\begin{array}{l}\text { I: } 65 \text { (SD 9) } \\
\text { C: } 63(10)\end{array}$ & $\begin{array}{l}\text { I: } 130 \\
\text { C: } 119\end{array}$ & RCT & $\begin{array}{l}48 \text { mths: } 6 \text { telephone calls: counsel, } \\
\text { answer questions, reviewed treatment at } 6 \text {, } \\
12,24 \& 36 \text { mths (case manager); dietician } \\
\text { or exercise specialist available; own } \\
\text { physician if required; participant \& } \\
\text { physician given progress report; TTM } \\
\text { based counselling. }\end{array}$ & $\begin{array}{l}\text { Usual care of } \\
\text { GP }\end{array}$ & $\begin{array}{l}\text { PA sessions, METs, diet, } \\
\text { HRQoL, WC, SBP, DBP, } \\
\text { smoking, LDL, HDL, } \\
\text { glucose, TC, TC/HDL, } \\
\text { TG, CVD risk }\end{array}$ & $\begin{array}{l}12,24,36 \& \\
48 \text { mths }\end{array}$ & $\begin{array}{l}\text { Telephone } \\
\text { calls; venue of } \\
\text { counselling } \\
\text { unclear }\end{array}$ \\
\hline $\begin{array}{l}\text { Lian et al. } \\
\text { (2014),[24] } \\
\text { China }\end{array}$ & $\begin{array}{l}\text { Patients with } \\
\text { CAD; } \\
\text { hospital }\end{array}$ & $\begin{array}{l}\text { Morning (I): } \\
64(\operatorname{SD} 9) \\
\text { Evening (I): } \\
62(10) \\
\text { C: } 61(10)\end{array}$ & $\begin{array}{l}\text { Morning }(\mathrm{I}) \text { : } \\
89 \\
\text { Evening }(\mathrm{I}) \text { : } \\
89 \\
\text { C: } 97\end{array}$ & 3 group $\mathrm{RCT}$ & $\begin{array}{l}12 \text { wks: Walk } \geq 30 \mathrm{~min} / \text { day on } \geq 5 \text { days/wk } \\
\text { at moderate intensity in morning or } \\
\text { evening; advice on smoking \& diet by } \\
\text { nutritionists; telephoned wkly to ensure } \\
\text { adherence. }\end{array}$ & $\begin{array}{l}\text { Maintain usual } \\
\text { level of PA. } \\
\text { Given advice } \\
\text { on smoking \& } \\
\text { diet. }\end{array}$ & $\begin{array}{l}\text { Walking \& total PA, TC, } \\
\text { LDL, HDL, TG, BMI, } \\
\text { weight, fibrinogen, white } \\
\text { blood cell count, platelet } \\
\text { count }^{\star \star *}\end{array}$ & 12 wks & $\begin{array}{l}\text { Telephone } \\
\text { calls }\end{array}$ \\
\hline $\begin{array}{l}\text { Mittag et } \\
\text { al. } \\
(2006),[43] \\
\text { Germany }\end{array}$ & $\begin{array}{l}\text { Patients with } \\
\text { MI, CABG or } \\
\text { PCl; hospital }\end{array}$ & $\begin{array}{l}\text { I: } 59 \text { (SD 10) } \\
\text { C: } 61 \text { (10) }\end{array}$ & $\begin{array}{l}\text { I: } 154 \\
\text { C: } 143\end{array}$ & $\begin{array}{l}\text { Prospective } \\
\text { RCT }\end{array}$ & $\begin{array}{l}12 \text { mths: Mthly nurse telephone contact } \\
\text { (mean of 10); counselling on PA, diet, } \\
\text { smoking, stress, psychosocial \& medical } \\
\text { problems; based on cognitive-behavioural } \\
\& \text { health psychology; posted } 6 \text { flyers on } \\
\text { general health. }\end{array}$ & $\begin{array}{l}6 \text { flyers on } \\
\text { general health } \\
\text { topics posted } \\
\text { every second } \\
\text { mth }\end{array}$ & $\begin{array}{l}\text { Anxiety, depression, } \\
\text { being active, diet, BMI, } \\
\text { TC, HDL, SBP, smoking, } \\
\text { diabetes, CVD risk }\end{array}$ & 12 mths & $\begin{array}{l}\text { Telephone } \\
\text { calls }\end{array}$ \\
\hline $\begin{array}{l}\text { Pinto et al. } \\
\text { (2011),[44] } \\
\text { USA }\end{array}$ & $\begin{array}{l}\text { Patients with } \\
\text { history of MI, } \\
\text { stable angina } \\
\text { \& CABG that } \\
\text { completed }\end{array}$ & $\begin{array}{l}\text { I: } 63 \text { (SD 9) } \\
\text { C: } 64(10)\end{array}$ & $\begin{array}{l}\text { I: } 44 \\
\text { C: } 52\end{array}$ & RCT & $\begin{array}{l}6 \text { mths: PA counselling based on TTM \& } \\
\text { SCT, using motivational interviewing via } \\
\text { telephone wkly over first } 2 \text { mths, biwkly for } \\
2 \text { mths mthly for } 2 \text { mths, by Coordinator; } \\
\text { self-monitoring (logs \& pedometer); posted }\end{array}$ & $\begin{array}{l}\text { Received calls } \\
\text { at same } \\
\text { intervals to } \\
\text { administer } \\
\text { symptom }\end{array}$ & $\begin{array}{l}\text { Primary: } \leq \text { moderate } \\
\text { PA/wk } \\
\text { Secondary: Motivational } \\
\text { readiness for exercise, }\end{array}$ & $6 \& 12 \mathrm{mths}$ & $\begin{array}{l}\text { Telephone } \\
\text { calls; posted } \\
\text { feedback } \\
\text { reports }\end{array}$ \\
\hline
\end{tabular}




\begin{tabular}{|c|c|c|c|c|c|c|c|c|c|}
\hline & CR & & & & $\begin{array}{l}\text { feedback reports at wks } 4,8,12,16 \& 20 ; \\
\text { posted printed materials on CVD health. }\end{array}$ & $\begin{array}{l}\text { questionnaire. } \\
\text { Posted printed } \\
\text { CVD info }\end{array}$ & $\begin{array}{l}\text { TC, LDL, HDL,CRP, } \\
\text { medication, peak VO } \\
\text { QoL(physical function) }\end{array}$ & & \\
\hline $\begin{array}{l}\text { Reid et al. } \\
\text { (2007),[28] } \\
\text { Canada }\end{array}$ & $\begin{array}{l}\text { Smokers with } \\
\text { CHD; hospital } \\
\text { inpatients }\end{array}$ & $\begin{array}{l}\text { I: } 54 \text { (SD 9) } \\
\text { C: } 53.9(9.0)\end{array}$ & $\begin{array}{l}\text { I: } 50 \\
\text { C: } 49\end{array}$ & $\begin{array}{l}2 \text { group pilot } \\
\text { study }\end{array}$ & $\begin{array}{l}8 \text { wks: Automated telephone calls } 3,14 \text { \& } \\
30 \text { days after discharge to assess smoking } \\
\text { status; additional counselling available from } \\
\text { nurse-specialist; pharmacotherapy } \\
\text { available. }\end{array}$ & $\begin{array}{l}\text { Usual care; } \\
\text { smoking } \\
\text { cessation } \\
\text { programme; } \\
\text { community } \\
\text { resources }\end{array}$ & Smoking & $12 \& 52$ wks & $\begin{array}{l}\text { Telephone } \\
\text { calls }\end{array}$ \\
\hline $\begin{array}{l}\text { Reid et al. } \\
\text { (2011),[38] } \\
\text { Canada }\end{array}$ & $\begin{array}{l}\text { Patients with } \\
\text { ACS not } \\
\text { accessing } \\
\text { CR; hospital } \\
\text { inpatients }\end{array}$ & $\begin{array}{l}\text { Overall: } 61 \\
\text { (SD 10) } \\
\text { I: } 60 \text { (10) } \\
\text { C: } 61(10)\end{array}$ & $\begin{array}{l}\text { I: } 69 \\
\text { C: } 72\end{array}$ & RCT & $\begin{array}{l}12 \text { mths: } 1 \text { face to face } \& 8 \text { telephone } \\
\text { contacts of motivational counselling with } \\
\text { physiotherapist: gaining commitment, } \\
\text { identifying valued outcomes, setting goals, } \\
\text { action planning, self-monitoring, identifying } \\
\text { opportunities for PA, problem solving, } \\
\text { feedback, encouragement, intensity } \\
\text { management \& links to medical care; } \\
\text { ecological perspective. }\end{array}$ & $\begin{array}{l}\text { Usual care } \\
\text { (Cardiology } \\
\text { Discharge } \\
\text { Book (health } \\
\text { info; walking } \\
\text { programme); } \\
\text { brief PA } \\
\text { advice from } \\
\text { cardiologist) } \\
\end{array}$ & $\begin{array}{l}\text { Distance travelled, } \\
\text { smoderate PA }\end{array}$ & $6 \& 12$ mths & $\begin{array}{l}\text { Telephone } \\
\text { calls; venue of } \\
\text { face-to-face } \\
\text { contact } \\
\text { unclear }\end{array}$ \\
\hline $\begin{array}{l}\text { Senuzun } \\
\text { et al. } \\
\text { (2006),[31] } \\
\text { Turkey }\end{array}$ & $\begin{array}{l}\text { Patients with } \\
\text { CHD; hospital } \\
\text { inpatients }\end{array}$ & $\begin{array}{l}\text { I: } 55 \text { (SD 8) } \\
\text { C: } 53(7)\end{array}$ & $\begin{array}{l}\text { I: } 30 \\
\text { C: } 30\end{array}$ & RCT & $\begin{array}{l}12 \text { wks: Written \& audio-visual education; } \\
\text { telephone call every } 2 \text { wks for self-efficacy } \\
\text { enhancing counselling sessions: reviewed } \\
\text { PA diary, walking goals, physiological } \\
\text { feedback \& social persuasion strategies. }\end{array}$ & OP care & $\begin{array}{l}\text { METs, exercise } \\
\text { tolerance, SEE, TC, TG, } \\
\text { HDL, LDL, BMI, SBP, } \\
\text { DBP }\end{array}$ & $12 \mathrm{wks}$ & $\begin{array}{l}\text { Telephone } \\
\text { calls; delivery } \\
\text { of materials } \\
\text { unclear }\end{array}$ \\
\hline $\begin{array}{l}\text { Wister et } \\
\text { al. } \\
\text { (2007),[55] } \\
\text { Canada }\end{array}$ & $\begin{array}{l}\text { Patients; } \\
\text { recruited by } \\
\text { GPs; poster, } \\
\text { newspaper } \\
\text { and radio } \\
\text { adverts }\end{array}$ & $\begin{array}{l}\text { Secondary } \\
\text { prevention } \\
\text { group } \\
\text { (I): } 57 \text { (SD 5) } \\
\text { C: } 57 \text { (5) }\end{array}$ & $\begin{array}{l}\text { Secondary } \\
\text { prevention } \\
\text { group (I): } \\
153 \\
\text { C: } 143\end{array}$ & 3 group $\mathrm{RCT}$ & $\begin{array}{l}12 \text { mths: Annual health report card posted; } \\
\text { Telehealth counselling } 6 \text { mthly from } 2 \\
\text { clinical lifestyle counsellors on smoking, } \\
\text { PA, diet \& stress; summaries of counselling } \\
\text { sessions \& educational materials posted. }\end{array}$ & Usual care & $\begin{array}{l}\text { Primary: Global CVD risk } \\
\text { Secondary: PA sessions, } \\
\text { health confidence, } \\
\text { perceived stress, diet, } \\
\text { TC, HDL, HRQoL, } \\
\text { glucose, SBP, smoking, } \\
\text { BMI, WC. }\end{array}$ & $12 \mathrm{mths}$ & $\begin{array}{l}\text { Telephone } \\
\text { calls, posted } \\
\text { report cards \& } \\
\text { printed info }\end{array}$ \\
\hline $\begin{array}{l}\text { Wu et al. } \\
\text { (2006),[52] } \\
\text { Taiwan }\end{array}$ & $\begin{array}{l}\text { Male patients } \\
\text { who had a } \\
\text { CABG; } \\
\text { referred by } \\
\text { surgeons }\end{array}$ & $\begin{array}{l}\text { Home-based } \\
\text { exercise (I): } \\
61(\text { SD 8) } \\
\text { C: } 62(10)\end{array}$ & $\begin{array}{l}\text { Home based } \\
\text { exercise (I): } \\
18 \\
\text { C: } 18\end{array}$ & 3 group $\mathrm{RCT}$ & $\begin{array}{l}12 \text { wks: tailored PA programme; updated } \\
\text { by office/ telephone consultation every } 2 \\
\text { wks by rehabilitation nurses; advised to } \\
\text { exercise } \geq 3 \text { times a wk. }\end{array}$ & $\begin{array}{l}\text { Normal levels } \\
\text { of PA }\end{array}$ & $\begin{array}{l}\text { Primary: HR recovery } \\
\text { Secondary: Resting \& } \\
\text { peak HR, workload, peak } \\
\mathrm{VO}_{2}\end{array}$ & 12 wks & $\begin{array}{l}\text { Telephone } \\
\text { calls or, } \\
\text { 'office' } \\
\text { consultation }\end{array}$ \\
\hline $\begin{array}{l}\text { Yates et } \\
\text { al. } \\
(2005),[56] \\
\text { USA }\end{array}$ & $\begin{array}{l}\text { Patients with } \\
\text { CABG or MI } \\
\text { who had } \\
\text { undergone } \\
\text { CR }\end{array}$ & $\begin{array}{l}\text { Average age } \\
\text { for each } \\
\text { group not } \\
\text { given. }\end{array}$ & $\begin{array}{l}\text { Telephone } \\
\text { (I): } 23 \\
\text { C: } 18\end{array}$ & 3 group RCT & $\begin{array}{l}9 \text { wks: CR booster sessions delivered by } \\
\text { nurse at } 3 \& 9 \text { wks via telephone: praised, } \\
\text { encouraged, discussed barriers to goals; } \\
\text { guided by Bandura's self-efficacy theory. }\end{array}$ & $\begin{array}{l}\text { Usual care (1 } \\
\text { telephone call } \\
\text { at } 4-6 \text { wks to } \\
\text { assess } \\
\text { satisfaction \& } \\
\text { risk reduction) }\end{array}$ & $\begin{array}{l}\text { Physical function (QoL); } \\
\text { PA sessions; HR, SBP \& } \\
\text { DBP }\end{array}$ & $3 \& 6$ mths & $\begin{array}{l}\text { Telephone } \\
\text { calls }\end{array}$ \\
\hline
\end{tabular}




\begin{tabular}{|c|c|c|c|c|c|c|c|c|c|}
\hline $\begin{array}{l}\text { Antypas \& } \\
\text { Wangberg } \\
(2014),[48] \\
\text { Norway }\end{array}$ & $\begin{array}{l}\text { Patients with } \\
\text { CVD (majority } \\
\text { MI); referred } \\
\text { by GP }\end{array}$ & $\begin{array}{l}\text { Intervention } \\
(\mathrm{I}): 60 \\
\text { Control }(\mathrm{C}) \text { : } \\
59\end{array}$ & $\begin{array}{l}\text { I: } 7 \\
8 \text { clusters } \\
\text { C: } 12 \\
10 \text { clusters }\end{array}$ & $\begin{array}{l}2 \text { group } \\
\text { cluster RCT }\end{array}$ & $\begin{array}{l}\text { Duration unclear. Tailored content on } \\
\text { website \& forum; messages via website \& } \\
\text { text: plan training activities/set wkly goals \& } \\
\text { remind of activities \& to text post-activity; } \\
\text { online graph feedback. }\end{array}$ & $\begin{array}{l}\text { Generic } \\
\text { website; info } \\
\text { on CR, } \\
\text { discussion } \\
\text { forum, activity } \\
\text { calendar }\end{array}$ & $\begin{array}{l}\text { Primary: Total PA } \\
\text { Secondary: SEE, social } \\
\text { support, depression, } \\
\text { anxiety, stage of change }\end{array}$ & $1 \& 3$ mths & $\begin{array}{l}\text { Website; } \\
\text { online forum } \\
\text { with text \& } \\
\text { email } \\
\text { reminders }\end{array}$ \\
\hline $\begin{array}{l}\text { Blasco et } \\
\text { al. } \\
(2012),[34] \\
\text { Spain }\end{array}$ & $\begin{array}{l}\text { Patients with } \\
\text { ACS; } \geq 1 \text { CVD } \\
\text { risk factor; } \\
\text { hospital }\end{array}$ & $\begin{array}{l}\text { I: } 61 \text { (SD 12) } \\
\text { C: } 61 \text { (12) }\end{array}$ & $\begin{array}{l}\text { I: } 87 \\
\text { C: } 83\end{array}$ & $\mathrm{RCT}$ & $\begin{array}{l}12 \text { mths: Tele-monitoring, } 3 \text { visits to } \\
\text { cardiologist, written \& verbal info on CVD } \\
\text { prevention; sent feedback on outcomes via } \\
\text { text message. }\end{array}$ & $\begin{array}{l}3 \text { visits to } \\
\text { cardiologist, } \\
\text { written \& } \\
\text { verbal CVD } \\
\text { prevention info } \\
\end{array}$ & $\begin{array}{l}\text { Smoking, LDL, SBP, } \\
\text { DBP, Hb1A, BMI, } \\
\text { HRQoL, anxiety }\end{array}$ & 12 mth & $\begin{array}{l}\text { Text message } \\
\& \text { web-based } \\
\text { tele- } \\
\text { monitoring; } \\
\text { clinic visits } \\
\end{array}$ \\
\hline \multicolumn{10}{|c|}{ Printed materials based delivery } \\
\hline $\begin{array}{l}\text { Sniehotta } \\
\text { et al. } \\
\text { (2005),[32] } \\
\text { Germany }\end{array}$ & $\begin{array}{l}\text { Patients with } \\
\text { CHD, } \\
\text { recruited } \\
\text { during } \\
\text { inpatient CR } \\
\text { treatment }\end{array}$ & $\begin{array}{l}\text { Overall: } 58 \\
\text { (SD 10) }\end{array}$ & Not stated & 3 group $\mathrm{RCT}$ & $\begin{array}{l}6 \text { wks: Both intervention groups given } \\
\text { individual planning session, using SCT, } \\
\text { before discharge. } \\
\text { Planning group: booklet for action \& coping } \\
\text { plans; Personalized wkly diary: one group } \\
\text { also posted } 6 \text { wkly diaries; tailored } \\
\text { personal plans. }\end{array}$ & Usual care & $\begin{array}{l}\text { Behavioural intentions, } \\
\text { SEE, action \& coping } \\
\text { planning, general PA, } \\
\text { strenuous PA }\end{array}$ & $\begin{array}{l}2 \& 6 \text { mths } \\
\text { after } \\
\text { discharge }\end{array}$ & $\begin{array}{l}\text { Printed } \\
\text { materials. } \\
\text { One group } \\
\text { posted diaries }\end{array}$ \\
\hline $\begin{array}{l}\text { Wolkanin- } \\
\text { Bartnik et } \\
\text { al. } \\
\text { (2011),[46] } \\
\text { Poland }\end{array}$ & $\begin{array}{l}\text { Patients with } \\
\text { acute Ml; OP } \\
\text { clinic }\end{array}$ & Overall: 70 & $\begin{array}{l}\text { I: } 59 \\
\text { C: } 56\end{array}$ & $\mathrm{RCT}$ & $\begin{array}{l}3 \text { mth intervention: Exercise guidebooks \& } \\
\text { diaries; offered phone consultations with } \\
\text { doctor. }\end{array}$ & OP care & $\begin{array}{l}\text { PA tolerance, resting \& } \\
\text { exercise HR, SBP, DBP, } \\
\text { peak workload, HR } \\
\text { recovery; CVD events, } \\
\text { mortality, admissions; } \\
\text { leisure time PA } \\
\end{array}$ & $3 \& 12$ mths & $\begin{array}{l}\text { Printed } \\
\text { materials; } \\
\text { option of } \\
\text { telephone } \\
\text { calls }\end{array}$ \\
\hline \multicolumn{10}{|c|}{ Home visit based delivery } \\
\hline $\begin{array}{l}\text { Goodman } \\
\text { et al. } \\
\text { (2008),[53] } \\
\text { England }\end{array}$ & $\begin{array}{l}\text { Patients on } \\
\text { waiting list for } \\
\text { CABG with at } \\
\text { least one } \\
\text { poorly } \\
\text { controlled risk } \\
\text { factor }\end{array}$ & $\begin{array}{l}\text { I: } 64 \\
\text { C: } 66\end{array}$ & $\begin{array}{l}\text { I: } 94 \\
\text { C: } 94\end{array}$ & $\mathrm{RCT}$ & $\begin{array}{l}12 \text { mths (mean): Mthly appt with cardiac } \\
\text { homecare nurse, assessed cardiac risk, } \\
\text { counselling (motivational interviewing } \\
\text { based on TTM); given manual; could } \\
\text { telephone between visits. Baseline appt } \\
\text { pre-surgery (mean wait } 9 \text { mths). }\end{array}$ & $\begin{array}{l}\text { Usual care } \\
\text { (hospital } \\
\text { helpline } \\
\text { telephone } \\
\text { numbers \& } \\
\text { pre-surgery } \\
\text { info day) }\end{array}$ & $\begin{array}{l}\text { Primary: Anxiety, } \\
\text { depression, TC, HDL, } \\
\text { SBP, DBP, BMI, length of } \\
\text { inpatient stay } \\
\text { Secondary: Smoking, } \\
\text { HRQoL, blood glucose }\end{array}$ & $\begin{array}{l}\text { 3 \& } 6 \text { mths } \\
\text { after } \\
\text { baseline } \\
\text { (unless } \\
\text { CABG); } \\
\text { admission \& } \\
3 \text { mths post } \\
\text { CABG } \\
\end{array}$ & $\begin{array}{l}\text { Home visits; } \\
\text { printed info; } \\
\text { option of } \\
\text { telephone } \\
\text { calls }\end{array}$ \\
\hline $\begin{array}{l}\text { Logan et } \\
\text { al. } \\
\text { (2009),[42] } \\
\text { Northern } \\
\text { Ireland }\end{array}$ & $\begin{array}{l}\text { Patients with } \\
\text { recent Ml or } \\
\text { unstable } \\
\text { angina; } \\
\text { Hospital } \\
\text { Cardiology } \\
\text { Directorate }\end{array}$ & $\begin{array}{l}\text { Nutritional } \\
\text { counselling } \\
\text { (I): } 58 \text { (SD 8) } \\
\text { Behavioural } \\
\text { counselling } \\
\text { (I): } 58(9)\end{array}$ & $\begin{array}{l}\text { Nutritional } \\
\text { counselling } \\
\text { (I): } 14 \\
\text { Behavioural } \\
\text { counselling } \\
\text { (I): } 10\end{array}$ & 3 group $\mathrm{RCT}$ & $\begin{array}{l}6 \text { mths: Nutritional counselling (I): Diet } \\
\text { advice \& sheet (health benefits, recipes \& } \\
\text { sample meal plan); home visit from dietitian } \\
\text { at wk } 1 \text { (optional) \& at mths } 1,2 \& 4 ; \\
\text { optional telephone contact with researcher. } \\
\text { Behavioural counselling (I): as above but } \\
\text { based on learning theory \& stage of }\end{array}$ & $\begin{array}{l}\text { Usual care } \\
\text { (conventional } \\
\text { dietary advice } \\
\text { \& diet sheet) }\end{array}$ & $\begin{array}{l}\text { Diet, stage of change, } \\
\text { vitamin C, oleic acid, } \\
\text { EPA }\end{array}$ & $6 \& 12$ mths & $\begin{array}{l}\text { Home visits; } \\
\text { printed info; } \\
\text { option of } \\
\text { telephone } \\
\text { calls }\end{array}$ \\
\hline
\end{tabular}




\begin{tabular}{|c|c|c|c|c|c|c|c|c|c|}
\hline & & C: 56 (11) & $\mathrm{C}: 12$ & & change model. & & & & \\
\hline $\begin{array}{l}\text { Sinclair et } \\
\text { al. } \\
(2005),[45] \\
\text { England }\end{array}$ & $\begin{array}{l}\text { Patients } \\
\text { hospitalised } \\
\text { with } \\
\text { suspected } \mathrm{Ml} \text {; } \\
\text { recruited on } \\
\text { admission }\end{array}$ & Not stated & $\begin{array}{l}\text { I: } 163 \\
\text { C: } 161\end{array}$ & RCT & $\begin{array}{l}\text { 6-8 wks: Nurse home-visits, } 1-2 \& 6-8 \text { wks } \\
\text { post discharge: guidance on risk factor } \\
\text { reduction \& activity; extra visits \& telephone } \\
\text { contacts if required; individualised info } \\
\text { booklet: } 6 \text {-wk PA programme, personal risk } \\
\text { factors, useful telephone numbers, advice. }\end{array}$ & $\begin{array}{l}\text { Usual care } \\
\text { (general } \\
\text { advice, OP } \\
\text { follow-up, } \\
\text { access to CR) }\end{array}$ & $\begin{array}{l}\text { Primary: HRQoL } \\
\text { Secondary: ADL, } \\
\text { mortality, hospital } \\
\text { readmissions, length of } \\
\text { inpatient stay, OP } \\
\text { attendances \& car driving }\end{array}$ & $\begin{array}{l}\text { Up to } 100 \\
\text { days after } \\
\text { baseline }\end{array}$ & $\begin{array}{l}\text { Home visits, } \\
\text { printed info; } \\
\text { option of } \\
\text { telephone } \\
\text { calls }\end{array}$ \\
\hline \multicolumn{10}{|c|}{ Home visit \& telephone based delivery } \\
\hline $\begin{array}{l}\text { Oerkild et } \\
\text { al. } \\
\text { (2012),[39] } \\
\text { Denmark }\end{array}$ & $\begin{array}{l}\text { Patients with } \\
\text { recent } \mathrm{MI} \text {, } \\
\text { PCI or CABG; } \\
\text { CVD } \\
\text { database }\end{array}$ & $\begin{array}{l}\text { I: } 77 \text { (SD 6) } \\
\text { C: } 77 \text { (8) }\end{array}$ & $\begin{array}{l}\text { I: } 19 \\
\text { C: } 21\end{array}$ & RCT & $\begin{array}{l}12 \text { mths: } 2 \text { home visits in } 6 \text { wk interval from } \\
\text { physiotherapist to develop PA programme, } \\
\text { telephone calls between visits to answer } \\
\text { questions; risk factor \& medical } \\
\text { management by cardiologist at baseline, } 3 \text {, } \\
6 \& 12 \text { mths; telephone calls at } 4 \& 5 \text { mths } \\
\text { by cardiologist to encourage PA; dietary } \\
\text { counselling \& smoking cessation offered. }\end{array}$ & $\begin{array}{l}\text { Usual care } \\
\text { (risk factor } \\
\text { intervention \& } \\
\text { medical } \\
\text { management } \\
\text { by } \\
\text { cardiologist) }\end{array}$ & $\begin{array}{l}\text { Primary: Exercise } \\
\text { capacity } \\
\text { Secondary: Lower limb } \\
\text { strength, PA (hrs; } \\
\text { intensity), TC, LDL,HDL, } \\
\text { DBP, SBP, smoking, } \\
\text { BMI, WHR, HRQoL, } \\
\text { anxiety, depression, } \\
\text { comorbidity, mortality, } \\
\text { admissions }\end{array}$ & $\begin{array}{l}3,6 \& 12 \\
\text { mths }\end{array}$ & $\begin{array}{l}\text { Home visits; } \\
\text { telephone } \\
\text { calls }\end{array}$ \\
\hline \multicolumn{10}{|c|}{ Method of delivery unclear } \\
\hline $\begin{array}{l}\text { Astengo et } \\
\text { al. } \\
(2010),[49] \\
\text { Sweden }\end{array}$ & $\begin{array}{l}\text { Patients with } \\
\text { stable angina } \\
\text { on waiting list } \\
\text { for } \mathrm{PCl}\end{array}$ & $\begin{array}{l}\text { I: } 62 \text { (SD 7) } \\
\text { C: } 65 \text { (SD 8) }\end{array}$ & $\begin{array}{l}\mathrm{I}: 28 \\
\mathrm{C}: 28\end{array}$ & $\mathrm{RCT}$ & $\begin{array}{l}8 \text { mths: Began } 2 \text { mths pre-PCl; bicycle } \\
\text { ergometer } \geq 30 \text { mins, } \geq 5 \text { days/wk, } \\
\text { resistance exercises \& mthly meetings with } \\
\text { physiotherapist to adjust intensity of } \\
\text { exercise \& motivate. }\end{array}$ & Usual care & $\begin{array}{l}\text { TC, LDL, HDL, TG, } \\
\text { HbA1c, CRP, interleukin, } \\
\text { serum amyloid, } 2 \mathrm{~h} \& \\
\text { fasting glucose, ApoA-1, } \\
\text { ApoB, HR, workload, } \\
\text { days \& sessions of PA }\end{array}$ & $\begin{array}{l}1 \text { wk pre } \\
\mathrm{PCl}, \\
3 \& 6 \text { mths } \\
\text { post } \mathrm{PCl}\end{array}$ & $\begin{array}{l}\text { Setting of } \\
\text { meetings } \\
\text { unclear }\end{array}$ \\
\hline \multicolumn{10}{|c|}{ Setting: General practice/primary care } \\
\hline $\begin{array}{l}\text { Adams et } \\
\text { al. } \\
\text { (2007),[40] } \\
\text { USA }\end{array}$ & $\begin{array}{l}\text { Participants } \\
\text { with } \geq 1 \text { : } \\
\text { angina, } \\
\text { CABG, CHF, } \\
\text { MI, PTCA, } \\
\text { stent, cathet- } \\
\text { erization }\end{array}$ & $\begin{array}{l}\text { Leap for life: } \\
62 \text { (SD 9) } \\
\text { CR } 61(10) \\
\text { Traditional } \\
\text { care (C): } 64 \\
(10)\end{array}$ & $\begin{array}{l}\text { Leap for Life } \\
\text { (I): } 25 \\
\text { CR (I): } 78 \\
\text { C: } 114\end{array}$ & 3 group $\mathrm{RCT}$ & $\begin{array}{l}\text { Leap for life: 8-hr workshop by MDT; given } \\
\text { workbook: info on CVD, medications, PA, } \\
\text { diet \& stress management. } \\
\text { CR: Medical evaluation, exercise training, } \\
\text { risk factor modification, education, } \\
\text { counselling; one meeting with social worker } \\
\text { \& dietician. }\end{array}$ & $\begin{array}{l}\text { Scheduled } \\
\text { visits, advised } \\
\text { on guidelines } \\
\text { for PA, } \\
\text { nutrition \& } \\
\text { medications }\end{array}$ & $\begin{array}{l}\text { TC, LDL, HDL, TC/HDL, } \\
\text { TG, HR, SBP, DBP, } \\
\text { weight, BMI, medication } \\
\text { use, depression, anxiety, } \\
\text { psychological distress, } \\
\text { ADL functional ability, } \\
\text { social isolation }\end{array}$ & $\begin{array}{l}3,6,9 \& 12 \\
\text { mths }\end{array}$ & $\begin{array}{l}\text { University } \\
\text { Medical } \\
\text { Centre; } \\
\text { printed info }\end{array}$ \\
\hline $\begin{array}{l}\text { Delaney et } \\
\text { al. } \\
(2008),[22]\end{array}$ & $\begin{array}{l}\text { Patients with } \\
\text { CHD; } \\
\text { general }\end{array}$ & Not stated & $\begin{array}{l}\text { I: } 673 \\
\text { C: } 670\end{array}$ & $\begin{array}{l}\text { Follow-up of } \\
\text { a RCT by } \\
\text { review of }\end{array}$ & $\begin{array}{l}12 \text { mths: Nurse-led clinic every } 2-6 \text { mths: } \\
\text { symptom \& drug review, BP, lipid \& } \\
\text { behavioural risk factor assessment; clinical }\end{array}$ & Usual care & $\begin{array}{l}\text { Total \& CVD mortality, } \\
\text { coronary events, hospital } \\
\text { admissions }\end{array}$ & $4 \& 10 \mathrm{yrs}$ & $\begin{array}{l}\text { General } \\
\text { practice; } \\
\text { printed info }\end{array}$ \\
\hline
\end{tabular}




\begin{tabular}{|c|c|c|c|c|c|c|c|c|c|}
\hline Scotland & practices & & & $\begin{array}{l}\text { national } \\
\text { datasets }\end{array}$ & $\begin{array}{l}\text { protocols \& record cards; leaflets on diet \& } \\
\text { programme to promote PA. }\end{array}$ & & & & \\
\hline $\begin{array}{l}\text { Krebs et } \\
\text { al. } \\
(2013),[37] \\
\text { New } \\
\text { Zealand }\end{array}$ & $\begin{array}{l}\text { Patients with } \\
\text { ACS \& hyper- } \\
\text { glycaemia }\end{array}$ & $\begin{array}{l}\text { Overall: } 63 \\
\text { (SD 12) }\end{array}$ & $\begin{array}{l}\text { I: } 14 \\
\text { C: } 15\end{array}$ & $\begin{array}{l}\text { Prospective } \\
\text { 9-mth } \\
\text { parallel } \\
\text { design RCT }\end{array}$ & $\begin{array}{l}9 \text { mths: nurse posted info: PA (green } \\
\text { prescription), smoking cessation \& diet; } \\
\text { attend GP at } 3,6 \& 9 \text { mths to optimise risk } \\
\text { factor management; GPs encouraged to } \\
\text { promote existing healthcare services; }\end{array}$ & GP follow-up & $\begin{array}{l}\text { Primary: Smoking, SBP, } \\
\text { DBP, TC, LDL,HDL, } \\
\text { TC/HDL, TG, BMI } \\
\text { Secondary: Weight, WC, } \\
\text { glucose \& HbA1c, } \\
\text { medication }\end{array}$ & 9 mths & $\begin{array}{l}\text { General } \\
\text { practice; } \\
\text { posted printed } \\
\text { info \& existing } \\
\text { healthcare } \\
\text { resources }\end{array}$ \\
\hline $\begin{array}{l}\text { Munoz et } \\
\text { al. } \\
\text { (2007),[33] } \\
\text { Spain }\end{array}$ & $\begin{array}{l}\text { Subjects with } \\
\text { MI, angina or } \\
\text { ischaemia } \\
\text { within } \\
\text { previous } 6 \text { yrs }\end{array}$ & $\begin{array}{l}\text { I: } 64 \text { (SD 10) } \\
\text { C: } 64(10)\end{array}$ & $\begin{array}{l}\text { I: } 378 \\
\text { C: } 340\end{array}$ & RCT & $\begin{array}{l}3 \text { yrs: Postal reminders to see GP every } 3 \\
\text { mths; GPs followed guidelines on CVD } \\
\text { prevention, provided advice on diet, PA \& } \\
\text { smoking cessation. }\end{array}$ & Usual care & $\begin{array}{l}\text { Primary: Total \& CVD } \\
\text { mortality, CVD events } \\
\text { Secondary: SBP, DBP, } \\
\text { TC, TG, LDL, HDL, } \\
\text { HRQoL, medications, } \\
\text { weight, BMI, glucose }\end{array}$ & $\begin{array}{l}3 \text { yrs or until } \\
\text { an endpoint } \\
\text { occurred }\end{array}$ & $\begin{array}{l}\text { General } \\
\text { Practice }\end{array}$ \\
\hline $\begin{array}{l}\text { Murphy et } \\
\text { al. } \\
\text { (2009),[26] } \\
\text { Ireland }\end{array}$ & $\begin{array}{l}\text { Patients with } \\
\text { CHD; general } \\
\text { practices }\end{array}$ & $\begin{array}{l}\text { I: } 69 \text { (SD 9) } \\
\text { C: } 67(10)\end{array}$ & $\begin{array}{l}\text { I: } 360 \\
\text { C: } 405\end{array}$ & Cluster RCT & $\begin{array}{l}18 \text { mths: GP \& nurse training in prescribing } \\
\text { \& behaviour change, administrative support } \\
\& \text { quarterly newsletter. Tailored care plans: } \\
\text { motivational interviewing, goal setting, info } \\
\text { booklet, 4-mthly review; based on SCT. }\end{array}$ & Usual care & $\begin{array}{l}\text { SBP, DBP, TC, total \& } \\
\text { CVD hospital admissions, } \\
\text { HRQoL, diet, smoking, } \\
\text { smoderate PA }\end{array}$ & 18 mths & $\begin{array}{l}\text { General } \\
\text { practice; } \\
\text { printed } \\
\text { materials }\end{array}$ \\
\hline $\begin{array}{l}\text { Ortega et } \\
\text { al. } \\
(2014),[47] \\
\text { Spain }\end{array}$ & $\begin{array}{l}\text { Low-risk } \\
\text { acute } \\
\text { coronary } \\
\text { patients; } \\
\text { primary care } \\
\text { \& hospital }\end{array}$ & $\begin{array}{l}\text { I: } 55 \text { (SD 11) } \\
\text { C: } 56(13)\end{array}$ & $\begin{array}{l}\mathrm{I}: 30 \\
\mathrm{C}: 44\end{array}$ & RCT & $\begin{array}{l}6 \text {-mths: Cycle ergometer exercise } \\
\text { programme supervised by primary care } \\
\text { nurses; } 3 \text { - } 5 \text { sessions/wk; appts s at } 4,10 \& \\
16 \text { wks with research physicians to } \\
\text { reinforce change in diet \& smoking. }\end{array}$ & $\begin{array}{l}\text { Usual care \& } \\
\text { guidelines on } \\
\text { unsupervised } \\
\text { walking } \\
\text { programme }\end{array}$ & $\begin{array}{l}\text { Primary: Peak } \mathrm{VO}_{2} \\
\text { Secondary: TC, HDL, } \\
\text { LDL, TG, SBP, DBP, HR } \\
\text { recovery, weight }\end{array}$ & $7 \mathrm{mths}$ & $\begin{array}{l}\text { Primary care } \\
\text { centres }\end{array}$ \\
\hline $\begin{array}{l}\text { Redfern et } \\
\text { al. (2009; } \\
2010),[19 \\
20] \\
\text { Australia }\end{array}$ & $\begin{array}{l}\text { ACS } \\
\text { survivors not } \\
\text { accessing } \\
\text { CR; recruited } \\
\text { as hospital } \\
\text { inpatients }\end{array}$ & $\begin{array}{l}\text { I: } 62(\mathrm{SD} 1) \\
\mathrm{C}: 67(1)\end{array}$ & $\begin{array}{l}\mathrm{I}: 67 \\
\mathrm{C}: 69\end{array}$ & RCT & $\begin{array}{l}3 \text { mths: GP consultation, } 5 \text { phone calls: risk } \\
\text { factor education, assertiveness training \& } \\
\text { lifestyle goal assessment; cholesterol } \\
\text { lowering module: healthy eating \& pharma } \\
\text { advice; choice of } 2 \text { other modules: BP } \\
\text { lowering, smoking cessation or PA; info } \\
\text { leaflets; pt selected preferred mgmt. option: } \\
\text { GP directed, hospital programme, } \\
\text { individual programme or self-help. }\end{array}$ & $\begin{array}{l}\text { Usual care } \\
\text { (pharmaco- } \\
\text { therapy \& } \\
\text { lifestyle } \\
\text { counselling) }\end{array}$ & $\begin{array}{l}\text { PA, smoking, TC, LDL, } \\
\text { HDL, TG, SBP, DBP, } \\
\text { BMI, medication, } \\
\text { depression, knowledge of } \\
\text { CVD risk factors, freq. of } \\
\text { medical consultations }\end{array}$ & 12 mths & $\begin{array}{l}\text { Tertiary } \\
\text { referral } \\
\text { hospital; } \\
\text { telephone } \\
\text { calls; printed } \\
\text { info; local } \\
\text { services }\end{array}$ \\
\hline \multicolumn{10}{|c|}{ Setting: Other } \\
\hline $\begin{array}{l}\text { Cohen et } \\
\text { al. } \\
\text { (2014),[35] } \\
\text { France }\end{array}$ & $\begin{array}{l}\text { Patients in } \\
\text { ICU for ACS; } \\
\geq 1 \text { education } \\
\text { modifiable }\end{array}$ & $\begin{array}{l}\text { I: } 58 \text { (SD 11) } \\
\text { C: } 56(11)\end{array}$ & $\begin{array}{l}\text { I: } 251 \\
\text { C: } 251\end{array}$ & $\begin{array}{l}2 \text { arm } \\
\text { parallel- } \\
\text { group RCT }\end{array}$ & $\begin{array}{l}12 \text { mths: Individual consultations (1, 2, 3,6, } \\
9 \text { \& } 12 \text { mths, with dietician): diet evaluation, } \\
\text { info, leaflets, discussion with partners; } \\
\text { nurse consultations for smokers: }\end{array}$ & $\begin{array}{l}\text { Usual care (1 } \\
\text { appt with } \\
\text { physician \& } \\
\text { cardiologist) }\end{array}$ & $\begin{array}{l}\text { Smoking, PA, weight, } \\
\text { WC, HRQoL, patient } \\
\text { knowledge, SBP, DBP, } \\
\text { LDL, HbA1c, medication, }\end{array}$ & 12 mths & $\begin{array}{l}\text { House of } \\
\text { Education }\end{array}$ \\
\hline
\end{tabular}




\begin{tabular}{|c|c|c|c|c|c|c|c|c|c|}
\hline & risk factor & & & & & & adverse events & & \\
\hline $\begin{array}{l}\text { Houle et } \\
\text { al. } \\
\text { (2011),[36] } \\
\text { Canada }\end{array}$ & $\begin{array}{l}\text { Patients } \\
\text { hospitalised } \\
\text { for ACS; } \\
\text { hospital } \\
\text { admissions } \\
\text { list }\end{array}$ & $\begin{array}{l}\text { I: } 58 \text { (SD 8) } \\
\text { C: } 59 \text { (9) }\end{array}$ & $\begin{array}{l}\text { I: } 32 \\
\text { C: } 33\end{array}$ & RCT & $\begin{array}{l}12 \text { mths: Self-monitoring (pedometer); PA } \\
\text { info before discharge; family member } \\
\text { invited; } 1 \text { phone call \& } 5 \text { face to face OP } \\
\text { consultations with nurse: barriers/solutions } \\
\text { to increase steps; medical care from own } \\
\text { physicians; based on SCT. }\end{array}$ & $\begin{array}{l}\text { Usual care } \\
\text { (health info, } \\
\text { access to CR, } \\
\text { follow-up by } \\
\text { physicians) }\end{array}$ & $\begin{array}{l}\text { Step count, smoking, } \\
\text { LDL, HDL, TC/HDL, TG, } \\
\text { fasting glucose, HR, } \\
\text { SBP, DBP, WC, SEE, } \\
\text { resting HR, ApoB }\end{array}$ & $\begin{array}{l}\text { PA at } 3,6,9 \\
\& 12 \text { mths. } \\
\text { Other } \\
\text { outcomes at } \\
6 \& 12 \text { mths }\end{array}$ & $\begin{array}{l}\text { OP setting; } \\
\text { irtelephone } \\
\text { call }\end{array}$ \\
\hline $\begin{array}{l}\text { Michalsen } \\
\text { et al. } \\
\text { (2006),[25] } \\
\text { Germany }\end{array}$ & $\begin{array}{l}\text { Patients with } \\
\text { CAD; hospital } \\
\text { inpatient stay }\end{array}$ & $\begin{array}{l}\text { I: } 59 \text { (SD 9) } \\
\text { C: } 60 \text { (9) }\end{array}$ & $\begin{array}{l}\text { I: } 48 \\
\text { C: } 53\end{array}$ & RCT & $\begin{array}{l}12 \text { mths: } 3 \text {-day retreat, then wkly 3-hr } \\
\text { meetings for } 10 \text { wks; then } 2 \text {-hr meetings } \\
\text { every other wk for } 9 \text { mths; programme } \\
\text { addressed: Mediterranean diet (individual } \\
\text { advice, group discussions \& cooking } \\
\text { classes), stress management (practice } \\
\text { techniques } \leq 30 \text { mins daily) \& PA. }\end{array}$ & $\begin{array}{l}\text { Usual care } \\
\text { with printed } \\
\text { advice }\end{array}$ & $\begin{array}{l}\text { Coronary calcification, } \\
\text { HF-HRV, baroreflex, TC, } \\
\text { LDL, HDL, TG, HR, BMI, } \\
\text { diet, SBP, DBP, angina, } \\
\text { HRQoL, medication, EE, } \\
\text { relaxation sessions, } \\
\text { smoking }\end{array}$ & 12 mths & $\begin{array}{l}\text { Non- } \\
\text { residential } \\
\text { retreat. } \\
\text { Setting of } \\
\text { follow-up } \\
\text { meetings } \\
\text { unclear }\end{array}$ \\
\hline $\begin{array}{l}\text { Pischke et } \\
\text { al. } \\
\text { (2008),[27] } \\
\text { USA }\end{array}$ & $\begin{array}{l}\text { Patients with } \\
\text { CAD }\end{array}$ & $\begin{array}{l}\text { I: } 57 \text { (SD 8) } \\
\text { C: } 59 \text { (10) }\end{array}$ & $\begin{array}{l}\text { I: } 19 \\
\text { C: } 16\end{array}$ & RCT & $\begin{array}{l}12 \text { mths: } 1 \text { wk retreat: Daily lectures by } \\
\text { clinical psychologist on diet, cooking } \\
\text { classes, grocery store tours, stress } \\
\text { management, aerobic exercise \& group } \\
\text { support meetings; patients' partners } \\
\text { invited; twice wkly group sessions for } 1 \mathrm{yr} \text {; } \\
\text { option to continue self-directed community. }\end{array}$ & Usual care & $\begin{array}{l}\text { Anxiety, depression, } \\
\text { social dysfunction, } \\
\text { insomnia, social support, } \\
\text { sense of coherence, } \\
\text { proneness to anger, type } \\
\text { A behaviour, PA, diet, } \\
\text { stress management }\end{array}$ & $1 \& 5$ yrs & $\begin{array}{l}\text { Retreat at } \\
\text { local hotel. } \\
\text { Setting of } \\
\text { follow-up } \\
\text { lectures } \\
\text { unclear. }\end{array}$ \\
\hline $\begin{array}{l}\text { Seki et al. } \\
\text { (2008),[30] } \\
\text { Japan }\end{array}$ & $\begin{array}{l}\text { Males with } \\
\text { CAD; } \\
\text { hospital OP } \\
\text { clinic }\end{array}$ & $\begin{array}{l}\text { I: } 69 \text { (SD 3) } \\
\text { C: } 70 \text { (4) }\end{array}$ & $\begin{array}{l}\text { I: } 18 \\
\text { C: } 16\end{array}$ & RCT & $\begin{array}{l}6 \text { mths: Wkly OP CR programme: } \\
\text { individualised exercise sessions, exercise } \\
\text { prescription ( } \geq 30 \text { min aerobic exercise } \\
\text { twice wkly at home); diet instruction; } \\
\text { education programme; individual } \\
\text { counselling by physicians \& nurses. }\end{array}$ & Usual care & $\begin{array}{l}\text { Step count, EE, peak } \\
\mathrm{VO}_{2} \text {, AT VO2, muscle } \\
\text { strength, flexibility, TC, } \\
\text { TG, HDL, LDL, BMI, WC, } \\
\text { glucose, body fat, lean } \\
\text { body weight, ApoA-I, } \\
\text { ApoB, HbA1c }\end{array}$ & 6 mths & Clinic-led \\
\hline
\end{tabular}

$\mathrm{Mth}=$ month, $\mathrm{Wk}=$ week, $\mathrm{yr}=$ year, mins = minutes, info = information, $\mathrm{CVD}=$ cardiovascular disease, $\mathrm{CABG}=$ coronary artery bypass grafting, $\mathrm{CAD}=\mathrm{coronary}$ artery disease, $\mathrm{TC}$ $=$ total cholesterol, $\mathrm{TC} / \mathrm{HDL}=$ total cholesterol $\mathrm{HDL}$ ratio, $\mathrm{LDL}=$ low-density lipoprotein, $\mathrm{HDL}=$ high-density lipoprotein, $\mathrm{HR}=\mathrm{heart}$ rate, $\mathrm{SBP}=\mathrm{systolic}$ blood pressure, $\mathrm{DBP}=$ diastolic blood pressure, $\mathrm{TG}=$ =triglycerides, $\mathrm{BMI}=$ body mass index, $\mathrm{PA}=$ physical activity, $\mathrm{HRQ}$ oL= Health-related quality of life, $\mathrm{HbA} 1 \mathrm{c}=$ glycated haemoglobin,

$\mathrm{Apo}=$ apolipoprotein, $\mathrm{SE}=$ self-efficacy, $\mathrm{SEE}=$ Self-efficacy expectation, $\mathrm{AT}=$ anaerobic threshold, $\mathrm{PCI}=$ percutaneous coronary intervention, $\mathrm{CR}=\mathrm{cardiac}$ rehabilitation, $\mathrm{EPA}=$ eicosapentaenoic acid, $A D L=$ activities of daily living, $E E=$ energy expenditure, $W C=$ waist circumference, freq. $=$ frequency, appt $=$ appointment, $p t=$ patient, $\mathrm{WHR}=\mathrm{waist}$ hip ratio, $\mathrm{CRP}=\mathrm{c}$ reactive protein, $\mathrm{TTM}=$ trans-theoretical model, $\mathrm{QALY}=$ quality adjusted life years, $\mathrm{OP}=$ out-patient

${ }^{*}$ Age shown to nearest whole number of years. ${ }^{* \star}$ Results for waist circumference divided into male \& female sub-groups. The Cochrane collaboration's instructions ${ }^{57}$ for combining two subgroups were followed. ${ }^{* \star}$ Results for the two intervention groups were combined using the Cochrane collaboration's instructions ${ }^{57}$ for all outcomes reported. 


\section{$1 \quad$ Risk of bias}

2 Overall, we judged six studies to be at high risk of bias (Table 2).[22,23,40,46,54,55]

3 Reasons for a judgement of high risk of bias included: lack of random sequence

4 generation, no blinding of personnel and/or outcome assessor, selective outcome

5 reporting and inappropriate use of assessments. We judged 21 studies to have a low 6 risk of bias and 11 as unclear risk of bias.

7

8

9

10

11

12

13

14

15

16

17

18

19

20 


\section{Table 2; Risk of bias of included studies}

\begin{tabular}{|l|l|l|l|l|l|l|l|}
\hline Study & $\begin{array}{l}\text { Random } \\
\text { sequence } \\
\text { generation } \\
\text { (selection } \\
\text { bias) }\end{array}$ & $\begin{array}{l}\text { Allocation } \\
\text { concealment } \\
\text { (selection } \\
\text { bias) }\end{array}$ & $\begin{array}{l}\text { Blinding of } \\
\text { participants, } \\
\text { personnel \& } \\
\text { outcome } \\
\text { assessors } \\
\text { (performance } \\
\text { \& detection } \\
\text { bias) }\end{array}$ & $\begin{array}{l}\text { Incomplete } \\
\text { outcome } \\
\text { data } \\
\text { (attrition } \\
\text { bias) }\end{array}$ & $\begin{array}{l}\text { Selective } \\
\text { reporting } \\
\text { (reporting } \\
\text { bias) }\end{array}$ & $\begin{array}{l}\text { Other } \\
\text { bias }\end{array}$ & $\begin{array}{l}\text { Overall } \\
\text { risk of } \\
\text { bias }\end{array}$ \\
& & & & \\
& & & & \\
\hline
\end{tabular}

\section{Setting: Participants homes}

\section{Internet based delivery}

\begin{tabular}{|c|c|c|c|c|c|c|c|}
\hline $\begin{array}{l}\text { Devi et al. } \\
(2014)[54]\end{array}$ & Low & Low & High & Low & High & Low & High \\
\hline $\begin{array}{l}\text { Lindsay et al. } \\
(2008, \\
2009)[18,17]\end{array}$ & Unclear & Unclear & Unclear & Unclear & Unclear & Unclear & Unclear \\
\hline $\begin{array}{l}\text { Reid et al. } \\
\text { (2012)[29] }\end{array}$ & Low & Low & Low & Low & Unclear & Low & Low \\
\hline \multicolumn{8}{|c|}{ Telephone based delivery } \\
\hline $\begin{array}{l}\text { Butler et al. } \\
(2009)[50]\end{array}$ & Low & Low & Unclear & Low & Unclear & Low & Low \\
\hline $\begin{array}{l}\text { Furber et al. } \\
(2010)[51]\end{array}$ & Low & Low & High & Low & Unclear & Low & Low \\
\hline $\begin{array}{l}\text { Hanssen et } \\
\text { al. }(2007)[41]\end{array}$ & Low & Low & Unclear & High & Unclear & Low & Low \\
\hline $\begin{array}{l}\text { Hawkes et al. } \\
\text { (2013);[16] } \\
\text { Turkstra et } \\
\text { al. (2013)[21] }\end{array}$ & Unclear & Unclear & Low & Low & High & Low & Low \\
\hline $\begin{array}{l}\text { Lear et al. } \\
(2006)[23]\end{array}$ & Low & Unclear & Unclear & High & Unclear & High & High \\
\hline $\begin{array}{l}\text { Lian et al. } \\
(2014)[24]\end{array}$ & Unclear & Unclear & Unclear & Low & High & Low & Unclear \\
\hline $\begin{array}{l}\text { Mittag et al. } \\
(2006)[43]\end{array}$ & Low & Low & Unclear & Low & Unclear & High & Low \\
\hline $\begin{array}{l}\text { Pinto et al. } \\
(2011)[44]\end{array}$ & Unclear & Unclear & Low & Low & High & Low & Low \\
\hline $\begin{array}{l}\text { Reid et al. } \\
\text { (2007)[28] }\end{array}$ & Low & Low & Unclear & Low & Unclear & Low & Low \\
\hline $\begin{array}{l}\text { Reid et al. } \\
(2011)[38]\end{array}$ & Low & Low & Low & High & Unclear & Low & Low \\
\hline $\begin{array}{l}\text { Senuzun et } \\
\text { al. (2006)[31] }\end{array}$ & Unclear & Unclear & Unclear & Unclear & Unclear & Low & Unclear \\
\hline $\begin{array}{l}\text { Wister et al. } \\
(2007)[55]\end{array}$ & Low & Low & Low & Low & Unclear & High & High \\
\hline $\begin{array}{l}\text { Wu et al. } \\
(2006)[52]\end{array}$ & Low & Unclear & Low & Low & Unclear & Low & Low \\
\hline $\begin{array}{l}\text { Yates et al. } \\
(2005)[56]\end{array}$ & Unclear & Unclear & Unclear & Unclear & Unclear & Low & Unclear \\
\hline \multicolumn{8}{|c|}{ Internet \& telephone based delivery } \\
\hline $\begin{array}{l}\text { Antypas and } \\
\text { Wangberg } \\
(2014)[48]\end{array}$ & Low & Low & Low & High & Unclear & Low & Low \\
\hline $\begin{array}{l}\text { Blasco et al. } \\
(2012)[34]\end{array}$ & Unclear & Unclear & Low & Low & Unclear & Low & Low \\
\hline
\end{tabular}




\begin{tabular}{|c|c|c|c|c|c|c|c|}
\hline \multicolumn{8}{|c|}{ Printed materials based delivery } \\
\hline $\begin{array}{l}\text { Sniehotta et } \\
\text { al. }(2005)[32]\end{array}$ & Unclear & Unclear & Unclear & High & Unclear & Low & Unclear \\
\hline $\begin{array}{l}\text { Wolkanin- } \\
\text { Bartnik et al. } \\
\text { (2011)[46] }\end{array}$ & Unclear & Unclear & Unclear & Unclear & High & Low & High \\
\hline \multicolumn{8}{|c|}{ Home visit based delivery } \\
\hline $\begin{array}{l}\text { Goodman et } \\
\text { al. (2008)[53] }\end{array}$ & Low & Unclear & Unclear & Low & Unclear & Low & Low \\
\hline $\begin{array}{l}\text { Logan et al. } \\
(2009)[42]\end{array}$ & Low & Unclear & Unclear & Low & Unclear & Low & Unclear \\
\hline $\begin{array}{l}\text { Sinclair et al. } \\
(2005)[45]\end{array}$ & Low & Low & Unclear & Low & Unclear & Low & Low \\
\hline \multicolumn{8}{|c|}{ Home visit \& telephone based delivery } \\
\hline $\begin{array}{l}\text { Oerkild et al. } \\
(2012)[39]\end{array}$ & Low & Low & Unclear & Low & Unclear & Low & Low \\
\hline \multicolumn{8}{|c|}{ Method of delivery unclear } \\
\hline $\begin{array}{l}\text { Astengo et } \\
\text { al. (2010)[49] }\end{array}$ & Unclear & Unclear & Unclear & Low & Unclear & Low & Unclear \\
\hline
\end{tabular}

\section{Setting: General practice/primary care}

\begin{tabular}{|l|l|l|l|l|l|l|l|}
\hline $\begin{array}{l}\text { Adams et al. } \\
\text { (2007)[40] }\end{array}$ & High & High & Unclear & High & Unclear & Unclear & High \\
\hline $\begin{array}{l}\text { Delaney et } \\
\text { al. (2008)[22] }\end{array}$ & Unclear & Unclear & Unclear & Low & Unclear & High & High \\
\hline $\begin{array}{l}\text { Krebs et al. } \\
(2013)[37]\end{array}$ & Unclear & Unclear & Unclear & Low & Unclear & Low & Unclear \\
\hline $\begin{array}{l}\text { Munoz et al. } \\
(2007)[33]\end{array}$ & Low & Unclear & Unclear & Low & High & Low & Low \\
\hline $\begin{array}{l}\text { Murphy et al. } \\
(2009)[26]\end{array}$ & Low & Low & Unclear & Low & Unclear & Low & Low \\
\hline $\begin{array}{l}\text { Ortega et al. } \\
(2014)[47]\end{array}$ & Unclear & Low & Unclear & Low & Unclear & Low & Unclear \\
\hline $\begin{array}{l}\text { Redfern et al. } \\
(2009, \\
2010)[19,20]\end{array}$ & Low & Low & Unclear & Low & Unclear & Low & Low \\
\hline
\end{tabular}

\section{Setting: Other}

\begin{tabular}{|l|l|l|l|l|l|l|l|}
\hline $\begin{array}{l}\text { Cohen et al. } \\
(2014)[35]\end{array}$ & Low & Low & Unclear & Low & Unclear & Low & Low \\
\hline $\begin{array}{l}\text { Houle et al. } \\
\text { (2011)[36] }\end{array}$ & Low & Unclear & Unclear & Low & Unclear & Unclear & Low \\
\hline $\begin{array}{l}\text { Michalsen et } \\
\text { al. (2006)[25] }\end{array}$ & Low & Low & Unclear & Low & Unclear & Low & Low \\
\hline $\begin{array}{l}\text { Pischke et al. } \\
\text { (2008)[27] }\end{array}$ & Unclear & Unclear & Unclear & High & Unclear & Low & Unclear \\
\hline $\begin{array}{l}\text { Seki et al. } \\
(2008)[30]\end{array}$ & Unclear & Unclear & Unclear & Low & Unclear & Low & Unclear \\
\hline
\end{tabular}

\section{Effectiveness of interventions}


Our primary outcome for meta-analysis was PA. We had planned that the primary

outcomes would include diet, smoking and alcohol behaviours, but we found insufficient data to include these in meta-analyses. We noted that several studies also reported biophysical outcomes, mental and physical health measures and total mortality; we decided to include them in our analyses in order to gain insight into the potential wider health benefits of the included studies. We included 31 studies (33 articles) in the meta-analysis: seven studies[18,24,28,32,38,42,48] were excluded due to insufficient data being available or outcomes that could not be pooled. For example, 21 different outcome measures were reported in the included studies (Appendix 2). Follow-up from baseline outcome assessment times were categorised into sub-groups of: three to six months, seven to 12 months and over 12 months.

\section{Physical Activity}

In total, eight studies reported a measure of PA that could be included in our metaanalyses. Our meta-analysis of three studies[29,30,36] (322 participants) (Appendix 3) showed a statistically significant increase in numbers of steps per week for intervention, compared to control groups (mean difference (MD) 7480 steps $(95 \% \mathrm{Cl}$ 1940, 13020)) (Table 3a). A further five studies found an increase in minutes of PA per week attributable to the intervention[16,27,44,50,51] (748 participants; MD 59.96 minutes $(95 \% \mathrm{Cl} 15.67,104.25))$ with moderate heterogeneity.

\section{Biophysical outcomes}

We found a statistically significant increase in peak $\mathrm{VO}_{2}$ for intervention groups compared to controls (4 studies;[30,44,47,52] 240 participants; MD $2.06 \mathrm{~mL} / \mathrm{kg} / \mathrm{min}$ $(95 \% \mathrm{Cl} 0.08,4.04))$ but with substantial heterogeneity.

For diastolic blood pressure (DBP), we found a statistically significant decrease for 
intervention groups compared to control groups (14 studies;[19,23,25,26,31,33,34,36,37,39,40,46,47,53] 2849 participants; MD -1.37 $(95 \% \mathrm{Cl}-2.52,-0.22))$, with moderate heterogeneity. However, for systolic blood pressure (SBP), outcomes for intervention and control group participants were not significantly different.

We found no statistically significant difference between intervention and control groups (12 studies;[23,25,30,31,33,34,37,39,40,50,53,55] 2103 participants) for BMI.

We found a small but statistically significant decrease in total cholesterol levels between the intervention and control groups (15 studies;[19,23,25,26,30,31,33,37,39,40,43,47,49,53,55] 3150 participants; MD -0.13 $(95 \% \mathrm{Cl}-0.25,-0.01))$ with moderate heterogeneity.

Our analysis of six studies[23,30,36,37,50,55] (752 participants) showed no significant difference in waist circumference for intervention groups compared to controls.

Mental and physical health measures

We found no statistically significant effects for mental health, based on either SF-12 (4 studies;[26,33,35,39] 1909 participants) or SF-36 (4 studies;[16,34,41,53] 877 participants) subscales, nor for physical health (SF-12: 4 studies;[26,33,35,39] 1909 participants; SF-36: 6 studies;[16,34,41,44,53,56] 1014 participants).

Total mortality

Five studies[22,29,33,39,45] (2913 participants) reported 638 deaths in total (intervention groups: 303 ; controls: 335$)$. The odds ratio (OR) for total mortality in intervention groups compared to controls was not significantly reduced. 
Table 3 (a); Results of initial meta-analyses

\begin{tabular}{|c|c|c|c|c|}
\hline & \multicolumn{4}{|c|}{ Meta-analyses } \\
\hline Outcomes & Number of trials & $\begin{array}{l}\text { Number of participants } \\
\text { in studies }\end{array}$ & $\begin{array}{c}\text { Effect size (MD; 95\% } \\
\text { Cl) }\end{array}$ & Heterogeneity $\left(\mathrm{I}^{2}, \%\right)$ \\
\hline $\begin{array}{l}\text { Physical activity } \\
\text { Steps per week } \\
\text { Minutes per week }\end{array}$ & $\begin{array}{l}3 \\
5\end{array}$ & $\begin{array}{l}322 \\
748\end{array}$ & $\begin{array}{c}7480(1940,13020)^{*} \\
59.96(15.67,104.25)^{\star}\end{array}$ & $\begin{array}{c}9 \\
47\end{array}$ \\
\hline Peak $\mathrm{VO}_{2}$ & 4 & 240 & $2.06(0.08,4.04)^{*}$ & 72 \\
\hline $\begin{array}{l}\text { Blood pressure } \\
\text { Diastolic } \\
\text { Systolic }\end{array}$ & $\begin{array}{l}14 \\
16 \\
\end{array}$ & $\begin{array}{l}2849 \\
3442 \\
\end{array}$ & $\begin{array}{c}-1.37(-2.52,-0.22)^{\star} \\
-1.79(-4.09,0.51)\end{array}$ & $\begin{array}{l}50 \\
70\end{array}$ \\
\hline Body Mass Index & 12 & 2103 & $-0.16(-0.62,0.31)$ & 27 \\
\hline Total cholesterol & 15 & 3150 & $-0.13(-0.25,-0.01)^{*}$ & 60 \\
\hline Total mortality & 5 & 2913 & $0.84(0.70,1.02)^{\star \star}$ & 0 \\
\hline $\begin{array}{l}\text { Mental wellbeing } \\
\text { SF } 12 \\
\text { SF } 36\end{array}$ & $\begin{array}{l}4 \\
4\end{array}$ & $\begin{array}{l}1909 \\
877\end{array}$ & $\begin{array}{c}-0.11(-0.96,0.74) \\
1.45(-0.00,2.90)\end{array}$ & $\begin{array}{l}0 \\
0\end{array}$ \\
\hline $\begin{array}{l}\text { Physical wellbeing } \\
\text { SF } 12 \\
\text { SF } 36\end{array}$ & $\begin{array}{l}4 \\
6\end{array}$ & $\begin{array}{l}1909 \\
1014\end{array}$ & $\begin{array}{l}0.50(-0.19,1.18) \\
1.36(-0.48,3.21)\end{array}$ & $\begin{array}{c}0 \\
43\end{array}$ \\
\hline Waist circumference & 6 & 752 & $-1.32(-4.02,1.38)$ & 43 \\
\hline
\end{tabular}

* Significant values

** Odds ratio 
1 Sensitivity analyses

2 Our first set of sensitivity analyses (Table $3 b$ ) that excluded studies[33,39,47,53]

3 which had only reported change in mean values for peak $\mathrm{VO}_{2}, \mathrm{DBP}, \mathrm{SBP}, \mathrm{BMI}$, total

4 cholesterol, and mental and physical health, and for which we had calculated follow-

5 up values, showed statistically significant improvements for intervention groups,

6 compared to controls, for DBP[33,39,47,53] (MD $-2.04 \mathrm{mmHg}(95 \% \mathrm{Cl}-3.37,-0.71)$ ),

7 SBP $33,39,47](\mathrm{MD}-3.14 \mathrm{mmHg}(95 \% \mathrm{Cl}-5.59,-0.69))$ and SF-36 mental health[53]

$8 \quad(M D 1.74(95 \%$ Cl 0.10, 3.38)) (Table 3b). However, we found no significant changes

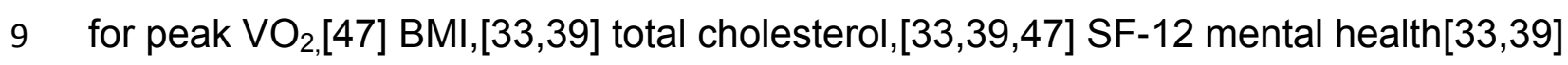

10 and physical health based on SF-12[33,39] and SF-36[53] outcomes.

11 We also conducted a second set of sensitivity meta-analyses, in which we excluded studies that we had determined were at high risk of bias overall.[22,23,40,46,54,55]

13 Excluded studies had reported data for DBP,[23,40,46] SBP, $[23,40,46,55]$

$14 \mathrm{BMI},[23,40,55]$ total cholesterol, $[23,40,55]$ waist circumference[23,55] and total

15 mortality.[22] These sensitivity analyses identified no statistically significant

16 outcomes.

17 Sensitivity analyses for PA was not required: all studies included in the meta-

18 analyses reported outcomes as absolute values and none were at high risk of bias

19 overall. 
Table 3 (b); Results of sensitivity analyses

\begin{tabular}{|c|c|c|c|c|c|c|c|c|}
\hline \multirow[b]{2}{*}{ Outcomes } & \multicolumn{4}{|c|}{$\begin{array}{c}\text { Sensitivity analyses } \\
\text { (excluding studies using substituted outcome data) }\end{array}$} & \multicolumn{4}{|c|}{$\begin{array}{c}\text { Sensitivity analyses } \\
\text { (excluding high risk of bias overall studies) }\end{array}$} \\
\hline & $\begin{array}{c}\text { No. } \\
\text { of trials }\end{array}$ & $\begin{array}{c}\text { No. of } \\
\text { Participants }\end{array}$ & $\begin{array}{c}\text { Effect size } \\
(\mathrm{MD} ; 95 \% \mathrm{Cl})\end{array}$ & $\begin{array}{l}\text { Heterogeneity } \\
\left(I^{2}, \%\right)\end{array}$ & $\begin{array}{c}\text { No. } \\
\text { of trials }\end{array}$ & $\begin{array}{c}\text { No. of } \\
\text { Participants }\end{array}$ & $\begin{array}{c}\text { Effect size } \\
(\mathrm{MD} ; 95 \% \mathrm{Cl})\end{array}$ & $\begin{array}{l}\text { Heterogeneity } \\
\left(1^{2}, \%\right)\end{array}$ \\
\hline $\begin{array}{l}\text { Physical activity } \\
\text { Steps per week } \\
\text { Minutes per week }\end{array}$ & $\begin{array}{l}N / A \\
N / A\end{array}$ & $\begin{array}{l}N / A \\
N / A\end{array}$ & $\begin{array}{l}N / A \\
N / A\end{array}$ & $\begin{array}{l}N / A \\
N / A\end{array}$ & $\begin{array}{l}N / A \\
N / A\end{array}$ & $\begin{array}{l}\mathrm{N} / \mathrm{A} \\
\mathrm{N} / \mathrm{A}\end{array}$ & $\begin{array}{l}N / A \\
N / A\end{array}$ & $\begin{array}{l}N / A \\
N / A\end{array}$ \\
\hline Peak $\mathrm{VO}_{2}$ & 3 & 166 & $1.70(-0.46,3.85)$ & 76 & N/A & N/A & N/A & $\mathrm{N} / \mathrm{A}$ \\
\hline $\begin{array}{l}\text { Blood pressure } \\
\text { Diastolic } \\
\text { Systolic }\end{array}$ & $\begin{array}{l}10 \\
11\end{array}$ & $\begin{array}{l}1829 \\
2126\end{array}$ & $\begin{array}{l}-2.04(-3.37,-0.71)^{\star} \\
-3.14(-5.59,-0.69)^{\star}\end{array}$ & $\begin{array}{l}47 \\
59\end{array}$ & $\begin{array}{l}11 \\
12\end{array}$ & $\begin{array}{l}2346 \\
2643\end{array}$ & $\begin{array}{l}-1.35(-2.73,0.02) \\
-1.62(-4.59,1.35)\end{array}$ & $\begin{array}{l}50 \\
73\end{array}$ \\
\hline Body Mass Index & 8 & 861 & $-0.08(-0.73,0.57)$ & 25 & 9 & 1419 & $-0.31(-0.71,0.10)$ & 0 \\
\hline Total cholesterol & 10 & 1834 & $-0.15(-0.32,0.02)$ & 69 & 12 & 2466 & $-0.11(-0.26,0.03)$ & 64 \\
\hline Total mortality & $\mathrm{N} / \mathrm{A}$ & $\mathrm{N} / \mathrm{A}$ & $N / A$ & $\mathrm{~N} / \mathrm{A}$ & 4 & 1570 & $0.79(0.53,1.18)^{\star *}$ & 0 \\
\hline $\begin{array}{l}\text { Mental wellbeing } \\
\text { SF } 12 \\
\text { SF } 36\end{array}$ & $\begin{array}{l}2 \\
3 \\
\end{array}$ & $\begin{array}{c}1151 \\
689 \\
\end{array}$ & $\begin{array}{l}0.35(-0.95,1.65) \\
1.74(0.10,3.38)^{*}\end{array}$ & $\begin{array}{l}0 \\
1\end{array}$ & $\begin{array}{l}N / A \\
N / A\end{array}$ & $\begin{array}{l}N / A \\
N / A\end{array}$ & $\begin{array}{l}N / A \\
N / A\end{array}$ & $\begin{array}{l}\text { N/A } \\
\text { N/A }\end{array}$ \\
\hline $\begin{array}{l}\text { Physical wellbeing } \\
\text { SF } 12 \\
\text { SF } 36\end{array}$ & $\begin{array}{l}2 \\
5\end{array}$ & $\begin{array}{c}1151 \\
826\end{array}$ & $\begin{array}{l}0.93(-0.54,2.40) \\
1.28(-1.04,3.60)\end{array}$ & $\begin{array}{l}35 \\
51\end{array}$ & $\begin{array}{l}N / A \\
N / A\end{array}$ & $\begin{array}{l}N / A \\
N / A\end{array}$ & $\begin{array}{l}N / A \\
N / A\end{array}$ & $\begin{array}{l}N / A \\
N / A\end{array}$ \\
\hline Waist circumference & 5 & 456 & $-1.32(-4.82,2.18)$ & 50 & 4 & 207 & $-3.09(-6.23,0.04)$ & 0 \\
\hline
\end{tabular}

* Significant values

** Odds ratio 
2 We were unable to conduct subgroup meta-analyses of the effect of different settings and components due to the variety of these within the studies included in this review.

4 However, 14 of the studies included in our meta-analysis reported statistically

5 significant improvements on intervention outcomes: eight of these studies were

6 implemented in participants' homes,[16,23,31,44,46,51,52,55] four in general

7 practice/primary care,[19,26,33,47] one at an outpatient setting[36] and one was

8 clinic-led.[30] Nine of these studies used telephone delivery of the

9 intervention.[16,19,23,31,36,44,51,52,55] Their interventions were based on the

Social Cognitive Theory,[16,26,36,51] the Transtheoretical Model,[23] and a combination of the Transtheoretical Model and Social Cognitive Theory.[44] All these interventions were individually tailored to participants; nine studies described a

psychological component, including counselling,[23,30,44,51,55] self-

monitoring,[36,51] goal setting,[26,31,36,51] motivational interviewing,[26,44] health coaching,[16] self-efficacy enhancing counselling sessions[31] and social persuasion strategies.[31] Only one study did not report using an education component[52] and six provided printed educational materials.[16,19,26,31,46,55] Eight studies focused on $\mathrm{PA}[30,31,36,44,46,47,51,52]$ and three used pedometers and diaries.[36,46,51]

In terms of overall risk of bias, we judged three of these studies to be of high risk of bias, $[23,46,55]$ three studies to be of unclear risk, $[30,31,47]$ and the eight other studies were deemed to be low risk. We found only three studies[16,44,52] to be low risk of bias for both outcome measurement and overall, reporting significant improvement for minutes of PA per week,[44] peak $\mathrm{VO}_{2}[52]$ and SF-36 mental[16] and physical health[44] subscales. 


\section{DISCUSSION}

Our study has shown that interventions for secondary CVD prevention, delivered in various venues within the community, can increase PA. This is important, as insufficient PA is a modifiable risk factor for CVD and premature mortality[58] and there is a need for effective approaches to prevention outside of traditional medical settings. Evidence for positive effects on peak $\mathrm{VO}_{2}$, blood pressure, total cholesterol and mental health was less clear. Interventions that reported effectiveness were delivered at home, general practice/primary care or outpatient settings, individualised, multicomponent and based on a theoretical framework. The relative effectiveness of interventions with different settings, or component designs or delivery modes could not be determined due to their heterogeneity.

Our initial meta-analyses showed a statistically significant improvement in peak $\mathrm{VO}_{2}$ among the intervention groups. However, there was substantial heterogeneity in the data and the sensitivity analyses excluding studies that reported outcome data as mean change from baseline, did not confirm this improvement. A previous systematic review[59] also found a significant improvement in peak $\mathrm{VO}_{2}$ for intervention participants but, this finding was based on a small number of studies.

We found total cholesterol to have a statistically significant decrease in the initial meta-analyses but this was not confirmed in the sensitivity analyses. This initial finding may be attributed to our use of data from studies that were excluded from the subsequent sensitivity analyses, which had a high risk of bias overall or for which we derived data inappropriately.

For DBP, our initial meta-analysis and the sensitivity analysis with exclusion of studies reporting outcome data as mean change from baseline, both found 
statistically significant decreases. However, this was not confirmed when studies with high risk of bias were removed, two of which had reported a statistically significant effect.

For DBP, SBP and SF-36 mental health subscale, initial meta-analyses showed no statistically significant effects but the sensitivity analyses excluding studies reporting outcome data as change in mean from baseline showed significant improvement.

The substituted data used initially may have hidden a true positive effect of the interventions but the sensitivity analyses included fewer participants, so results must be interpreted with caution. Given the contradictory findings between SF-12 and SF36 for mental health outcomes and that previous literature has shown that SF-12 and SF-36 are comparable measures,[60] there is a need for further study data to allow conclusive evaluation of these effects of community-based interventions.

Relevant other outcomes, such as behaviour change relating to diet, smoking and alcohol use, or health service usage, hospital admissions and CVD events could not be included in the meta-analysis. For many studies included in our review these outcomes were not reported; for others, the multiplicity of different outcome measures used prevented pooling of data for analyses.

Nineteen studies which were identified as being eligible for inclusion in our review used internet and/or telephone as an intervention component. Fourteen of these studies were included in our meta-analyses and were found to contribute to significant changes in PA behaviour. This interest in technology for CVD prevention is justified: $86 \%$ of households (22.5 million) in Great Britain have internet access[61] and previous systematic reviews, focused on telephone/internet CVD prevention interventions, reported favourable outcomes. $[9,11]$ This review adds to that evidence 
base: one previous review[9] included only interventions delivered primarily by internet and the other[11] included both internet and telephone delivery but focused on primary CVD prevention.

In concordance with NICE recommendations[62], the majority of studies included in our review were multicomponent. Further, previous systematic reviews, one focused on home-based programmes for secondary CVD prevention and the other focused on $\mathrm{PA}[7,63]$ also found that effective healthy behaviour change interventions were those combining multiple components such as education, engagement in PA and psychological support. This systematic review differs from those reviews by examining the effectiveness of interventions, involving different modes of delivery and different community venues, for behaviour change in secondary CVD prevention.

\section{Limitations}

Since the majority of included studies evaluated complex multifactorial interventions, we could not determine the independent contributions of different intervention components or optimal combinations. Differing content of control conditions across trials resulted in difficulty deciding if some were 'minimal intervention'. We did not limit our inclusion criteria to include only randomised studies but also included studies which reported other designs with control groups and interrupted time series. Though randomised study designs are considered most robust and can be used to infer causation, we recognise that that these are not always appropriate to address questions related to community-based interventions, especially health system interventions or implementation strategies.[64] Our exclusion of simple pre- and post-intervention studies may have resulted in the exclusion of relevant uncontrolled 
97 before and after studies that may have increased the applicability of the results and extended the number of settings included[65]. In planning future updates to this review and those with similar scope, full consideration needs to be given to including a wider range of study designs. Also, different countries use a variety of terms for healthcare venues, so some settings may have been categorised inappropriately. The majority of follow-up times were less than 12 months and there was limited availability of longer-term data, so long-term behaviour change could not be determined.

The heterogeneity of measurements reported by studies limited the number of outcomes for inclusion in our meta-analyses: future research should plan to include standardised and objective outcome measures that have been reported previously in order to allow further meta-analyses and provide conclusive evidence to inform planning of services. We found scant detail on the content, setting, bias potential, theoretical foundation of interventions and on outcomes relating to health service utilisation and cost. Despite socio-economic position being negatively associated with healthy lifestyle behaviours,[66] only one study focused on socio-economically deprived communities, thus there is a need for further study of this sub-group.

Similar to previous work,[7] we found few female participants in studies: greater efforts to include women would allow findings to be more generalisable.

\section{CONCLUSION}

This novel review provides evidence for the effectiveness of a variety of secondary CVD prevention programmes, delivered in venues within the community on modification of behavioural risk factors and highlights their positive effects on PA, peak VO2, blood pressure, total cholesterol and mental health. Healthcare 
121 professionals may recommend participation in interventions that are based on

122 theoretical frameworks, tailored to individuals and delivered in community-based

123 settings to promote reduction of CVD risk.

\section{Contributions}

125 All authors were responsible for study conception, design and initial search strategy.

126 ERL and DTB carried out the independent screening of articles, data extraction and

127 quality assessments. ERL was responsible for data analysis and for drafting the

128 manuscript. MEC and MAT provided consensus for inclusion of articles and

129 differences in extracted data. All authors contributed to manuscript revisions. All

130 authors read and approved the final manuscript.

\section{Conflict of interest}

132 The authors declare that there are no conflicts of or competing interests.

\section{Funding}

134 This work was carried out as part of a PhD funded by the UKCRC Centre of

135 Excellence for Public Health (Northern Ireland).

\section{REFERENCES}

137 1. Wang $H$, Naghavi M, Allen $\mathrm{C}$, et al. Global, regional, and national life expectancy,

138 all-cause mortality, and cause-specific mortality for 249 causes of death, 1980-2015:

139 a systematic analysis for the Global Burden of Disease Study 2015. Lancet

140 2016;388(10053):1459-1544.doi:10.1016/S0140-6736(16)31012-1

2. Vos T, Allen C, Arora M, et al. 2016. Global, regional, and national incidence, prevalence, and years lived with disability for 310 diseases and injuries, 1990-2015: 
a systematic analysis for the Global Burden of Disease Study 2015. Lancet 2016;388(10053):1545-602.doi:10.1016/S0140-6736(16)31678-6

3. Wilkins E, Wilson L, Wickramasinghe K, et al. European cardiovascular disease statistics 2017. Brussels: European Heart Network. Available: file://Q:/europeancardiovascular-disease-statistics-2017\%20(1).pdf

4. Doherty $\mathrm{P}$, Petre $\mathrm{C}$, Onion $\mathrm{N}$, et al. The national audit of cardiac rehabilitation annual statistical report 2015. London: British Heart Foundation. Available: http://www.cardiacrehabilitation.org.uk/docs/BHF_NACR_Report_2015.pdf

\section{De Vos C, Li X, Van Vlaenderen I, et al. Participating or not in a cardiac} rehabilitation programme: Factors influencing a patient's decision. Eur J Prev Cardiol 2013;20(2):341-8.doi:10.1177/2047487312437057
6. Kotseva K, Wood D, De Bacquer D, et al. EUROASPIRE IV: A European society of cardiology survey on the lifestyle, risk factor and therapeutic management of coronary patients from 24 European countries. Eur J Prev Cardiol 2016;23(6):63648.doi:10.1177/2047487315569401

7. Clark AM, Haykowsky M, Kryworuchko J, et al. A meta-analysis of randomized control trials of home-based secondary prevention programs for coronary artery disease. Eur J of Cardiovasc Prev Rehabil 2010;17(3):261-

70.doi:10.1097/HJR.0b013e32833090ef

8. Devi R, Singh SJ, Powell J, et al. Internet-based interventions for the secondary prevention of coronary heart disease. Cochrane Database Syst Rev 2015;(12).doi:10.1002/14651858.CD009386.pub2

9. Barth J, Jacob T, Daha I, et al. Psychosocial interventions for smoking cessation in patients with coronary heart disease. Cochrane Database Syst Rev 2015;(7).doi:10.1002/14651858.CD006886.pub2 
10. Neubeck L, Redfern J, Fernandez R, et al. Telehealth interventions for the secondary prevention of coronary heart disease: A systematic review. Eur $J$ of Cardiovasc Prev Rehabil 2009;16(3):281-9.doi:10.1097/HJR.0b013e32832a4e7a 11. Widmer RJ, Collins NM, Collins CS, et al. Digital health interventions for the prevention of cardiovascular disease: A systematic review and meta-analysis. Mayo Clin Proc 2015;90(4):469-80.doi:10.1016/j.mayocp.2014.12.026

12. Moher D, Liberati A, Tetzlaff J, et al. Preferred reporting items for systematic reviews and meta-analyses: The PRISMA statement. PLoS Medicine 2009;6(7):e1000097.doi:10.1371/journal.pmed.1000097

13. Baker PRA, Francis DP, Soares J, et al. Community wide interventions for increasing physical activity. Cochrane Database Syst Rev 2015;(1).doi:10.1002/14651858.CD008366.pub3

14. Ebrahim S, Taylor F, Ward K, et al. 341 Multiple risk factor interventions for primary prevention of coronary heart disease. Cochrane Database Syst Rev 2011;(1).doi:10.1002/14651858.CD001561.pub3

15. Higgins JPT, Altman DG, Gotzsche PC, et al. The cochrane collaboration's tool for assessing risk of bias in randomised trials. BMJ 2011;343:d5928.doi:10.1136/bmj.d5928

16. Hawkes AL, Patrao TA, Atherton J, et al. Effect of a telephone-delivered coronary heart disease secondary prevention program (proactive heart) on quality of life and health behaviours: Primary outcomes of a randomised controlled trial. Int $J$ Behav Med 2013;20(3):413-24.doi:10.1007/s12529-012-9250-5 
17. Lindsay S, Smith S, Bellaby P, et al. The health impact of an online heart disease support group: A comparison of moderated versus unmoderated support. Health Educ Res Aug 2009;24(4):646-654. doi: 10.1093/her/cyp001

18. Lindsay S, Bellaby P, Smith S, et al. Enabling healthy choices: is ICT the highway to health improvement? Health 2008;12(3):31331.doi:10.1177/1363459308090051

19. Redfern J, Briffa T, Ellis E, et al. Choice of secondary prevention improves risk factors after acute coronary syndrome: 1-year follow-up of the CHOICE (choice of health options in prevention of cardiovascular events) randomised controlled trial. Heart 2009;95(6):468-75.doi:10.1136/hrt.2008.150870

20. Redfern J, Menzies M, Briffa T, et al. Impact of medical consultation frequency on modifiable risk factors and medications at 12 months after acute coronary syndrome in the CHOICE randomised controlled trial. Int J Cardiol 2010;145(3):4816.doi:10.1016/j.ijcard.2010.04.035

21. Turkstra E, Hawkes AL, Oldenburg B, et al. Cost-effectiveness of a coronary heart disease secondary prevention program in patients with myocardial infarction: results from a randomised controlled trial (ProActive Heart). BMC Cardiovasc Disord 2013;13(33).doi:10.1186/1471-2261-13-33

22. Delaney EK, Murchie P, Lee AJ, et al. Secondary prevention clinics for coronary heart disease: A 10-year follow-up of a randomised controlled trial in primary care. Heart 2008;94(11):1419-23.doi:10.1136/hrt.2007.126144 
23. Lear SA, Spinelli JJ, Linden W, et al. The extensive lifestyle management intervention (ELMI) after cardiac rehabilitation: A 4-year randomized controlled trial. Am Heart J 2006;152(2):333-9.doi:10.1016/j.ahj.2005.12.023

24. Lian X, Zhao D, Zhu M, et al. The influence of regular walking at different times of day on blood lipids and inflammatory markers in sedentary patients with coronary artery disease. Prev Med 2014;58:64-9.doi:10.1016/j.ypmed.2013.10.020

25. Michalsen A, Knoblauch NT, Lehmann N, et al. Effects of lifestyle modification on the progression of coronary atherosclerosis, autonomic function, and angina-the role of GNB3 C825T polymorphism. Am Heart J 2006;151(4):870-

7.doi:10.1016/j.ahj.2005.06.025

26. Murphy AW, Cupples ME, Smith SM, et al. Effect of tailored practice and patient care plans on secondary prevention of heart disease in general practice: Cluster randomised controlled trial. BMJ 2009;339:b4220.doi:10.1136/bmj.b4220

27. Pischke CR, Scherwitz L, Weidner G, et al. Long-term effects of lifestyle changes on well-being and cardiac variables among coronary heart disease patients. Health Psychol 2008;27(5):584-92.doi:10.1037/0278-6133.27.5.584

28. Reid RD, Pipe AL, Quinlan B, et al. Interactive voice response telephony to promote smoking cessation in patients with heart disease: A pilot study. Patient Educ Couns 2007;66(3):319-26.doi:10.1016/j.pec.2007.01.005

29. Reid RD, Morrin LI, Beaton LJ, et al. Randomized trial of an internet-based computer tailored expert system for physical activity in patients with heart disease. Eur J Prev Cardiol 2012;19(6):1357-64.doi:10.1177/1741826711422988 
233 30. Seki E, Watanabe Y, Shimada K, et al. Effects of a phase III cardiac

234 rehabilitation program on physical status and lipid profiles in elderly patients with

235 coronary artery disease: Juntendo cardiac rehabilitation program (J-CARP).

236 Circulation Journal 2008;72(8):1230-4.doi:10.1253/circj.72.1230

237 31. Senuzun F, Fadiloglu C, Burke LE, et al. Effects of home-based cardiac exercise 238 program on the exercise tolerance, serum lipid values and self-efficacy of coronary 239 patients. Eur J Cardiovasc Prev Rehabil 2006;13(4):640-

240 5.doi:10.1097/01.hjr.0000198445.41680.ec

241 32. Sniehotta FF, Scholz U, Schwarzer R, et al. Long-term effects of two psychological interventions on physical exercise and self-regulation following coronary rehabilitation. Int J Behav Med 2005;12(4):24455.doi:10.1207/s15327558ijbm1204_5

33. Munoz MA, Vila J, Cabanero M, et al. Efficacy of an intensive prevention program in coronary patients in primary care, a randomised clinical trial. Int J Cardiol 2007;118(3):312-20.doi:10.1016/j.ijcard.2006.07.015

34. Blasco A, Carmona M, Fernandez-Lozano I, et al. Evaluation of a telemedicine service for the secondary prevention of coronary artery disease. J Cardiopulmon Rehabil Prev 2012;32(1):25-31.doi:10.1097/HCR.0b013e3182343aa7

251 35. Cohen A, Assyag P, Boyer-Chatenet L, et al. An education program for risk factor management after an acute coronary syndrome: A randomized clinical trial. 
36. Houle J, Doyon O, Vadeboncoeur N, et al. Innovative program to increase physical activity following an acute coronary syndrome: Randomized controlled trial. Patient Educ Couns 2011;85:e237-44.doi:10.1016/j.pec.2011.03.018

37. Krebs JD, Van Wissen KA, Harding SA, et al. An intervention trial for patients with hyperglycaemia and acute coronary syndrome: How effective is lifestyle advice? PCCJ 2013;6:72-5.doi:10.3132/pccj.2013.008

38. Reid RD, Morrin LI, Higginson LA, et al. Motivational counselling for physical activity in patients with coronary artery disease not participating in cardiac rehabilitation. Eur J of Prev Cardiol 2011;19(2):161-

6.doi:10.1177/1741826711400519

39. Oerkild B, Frederiksen M, Hansen JF, et al. Home-based cardiac rehabilitation is an attractive alternative to no cardiac rehabilitation for elderly patients with coronary heart disease: Results from a randomised clinical trial. BMJ Open 2012;2:e001820.doi:10.1136/bmjopen-2012-001820

40. Adams JL, Nuss T, Banks C, et al. Risk factor outcome comparison between exercise based cardiac rehabilitation, traditional care, and an educational workshop. Cardiac Rehabil Outcomes 2007;38(2):83-8.doi:10.3928/00220124-20070301-04

41. Hanssen TA, Nordrehaug JE, Eide GE, et al. Improving outcomes after myocardial infarction: A randomized controlled trial evaluating effects of a telephone follow-up intervention. Eur J Cardiovasc Prev Rehabil 2007;14:429-

37.doi:10.1097/HJR.0b013e32801da123

42. Logan KJ, Woodside JV, Young I, et al. Adoption and maintenance of a Mediterranean diet in patients with coronary heart disease from a northern European 
population: A pilot randomised trial of different methods of delivering Mediterranean

43. Mittag O, China $\mathrm{C}$, Hoberg $\mathrm{E}$, et al. Outcomes of cardiac rehabilitation with versus without a follow-up intervention rendered by telephone (Luebeck follow-up trial): Overall and gender-specific effects. Int J Rehab Res 2006;29:295302.doi:10.1097/MRR.0b013e328010ba9a

44. Pinto BM, Goldstein MG, Papandonatos GD, et al. Maintenance of exercise after phase II cardiac rehabilitation: A randomized controlled trial. Am J Prev Med 2011;41(3):274-83.doi:10.1016/j.amepre.2011.04.015

45. Sinclair AJ, Conroy SP, Davies M, et al. Post-discharge home-based support for older cardiac patients: A randomised controlled trial. Age Ageing 2005;34(4):33843.doi: 10.1093/ageing/afi116

46. Wolkanin-Bartnik J, Pogorzelska H, Bartnik A. Patient education and quality of home based rehabilitation in patients older than 60 years after acute myocardial infarction. J Cardiopulmon Rehabil Prev 2011;31(4):249-

47. Ortega R, Garcia-Ortiz L, Torcal J, et al. Supervised exercise for acute coronary patients in primary care: A randomized clinical trial. Fam Pract 2014;31(1):20-

48. Antypas K, Wangberg SC. An internet- and mobile-based tailored intervention to enhance maintenance of physical activity after cardiac rehabilitation: Short term results of a randomized controlled trial. J Med Internet Res 2014;16(3):e77.doi:10.2196/jmir.3132 
49. Astengo M, Dahl A, Karlsson T, et al. Physical training after percutaneous coronary intervention in patients with stable angina: Effects on working capacity, metabolism, and markers of inflammation. Eur J of Cardiovasc Prev Rehabil 2010;17(3):349-54.doi:10.1097/HJR.0b013e3283336c8d

50. Butler L, Furber S, Phongsavan P, et al. Effects of a pedometer-based intervention on physical activity levels after cardiac rehabilitation: A randomized controlled trial. J Cardiopulmon Rehabil Prev 2009;29(2):105-

14.doi:10.1097/HCR.0b013e31819a01ff

51. Furber S, Butler L, Phongsavan $\mathrm{P}$, et al. Randomised controlled trial of a pedometer based telephone intervention to increase physical activity among cardiac patients not attending cardiac rehabilitation. Patient Educ Couns 2010;80:2128.doi:10.1016/j.pec.2009.11.012

52. Wu S, Lin Y, Chen C, et al. Cardiac rehabilitation vs. home exercise after coronary artery bypass graft surgery. Am J Phys Med Rehabil 2006;85(9):7117.doi:10.1097/01.phm.0000228597.64057.66

53. Goodman H, Parsons A, Davison J, et al. A randomised controlled trial to evaluate a nurse-led programme of support and lifestyle management for patients awaiting cardiac surgery 'Fit for surgery: Fit for life' study. Eur J Cardiovasc Nurs 2008;7(3):189-95.doi:10.1016/j.ejcnurse.2007.11.001

54. Devi R, Powell J, Singh S. A web-based program improves physical activity outcomes in a primary care angina population: Randomized controlled trial. J Med Internet Res 2014;16(9):37-49.doi:10.2196/jmir.3340 
322 55. Wister A, Loewen N, Kennedy-Symonds H, et al. One-year follow-up of a therapeutic lifestyle intervention targeting cardiovascular disease risk. CMAJ 2007;177(8):859-65.doi:10.1503/cmaj.061059

56. Yates BC, Anderson T, Hertzog M, et al. Effectiveness of follow-up booster sessions in improving physical status after cardiac rehabilitation: Health, behavioral, and clinical outcomes. Appl Nurs Res 2005;18(1):5962.doi:10.1016/j.apnr.2004.06.012

57. Higgins JPT, Green S (editors). Cochrane Handbook for Systematic Reviews of Interventions. Version 5.1.0 [updated March 2011]. The Cochrane Collaboration 2011. Available: www.handbook.cochrane.org.

58. Lee I, Shiroma EJ, Lobelo F, et al. Effect of physical inactivity on major non communicable diseases worldwide: An analysis of burden of disease and life expectancy. Lancet 2012;380(9838):219-29.doi:10.1016/S0140-6736(12)61031-9

59. Cole JA, Smith SM, Hart N, et al. Systematic review of the effect of diet and exercise lifestyle interventions in the secondary prevention of coronary heart disease. Cardiol Res Pract 2010:232-351.doi:10.4061/2011/232351

60. Muller-Nordhorn J, Roll S, Willich SN. Comparison of the short form (SF)-12 health status instrument with the SF-36 in patients with coronary heart disease. Heart 2004;90:523-7.doi:10.1136/hrt.2003.013995

61. Office for National Statistics. Internet access - households and individuals: 2015. 2015. Available: 
meinternetandsocialmediausage/bulletins/internetaccesshouseholdsandindividuals/2 015-08-06\#computer-and-internet-use (accessed July 2017).

62. National Institute for Health and Care Excellence. Behaviour change: General approaches. 2007. Manchester, England: NICE.

63. Cleland CL, Tully MA, Kee F et al. The effectiveness of physical activity interventions in socio-economically disadvantaged communities: A systematic review. Prev Med 2012;54:371-80.doi:10.1016/j.ypmed.2012.04.004

64. Cochrane Effective Practice and Organisation of Care (EPOC). What study designs can be considered for inclusion in an EPOC review and what should they be called? EPOC Resources for review authors. 2017. Available:

\section{http://epoc.cochrane.org/resources/epoc-resources-review-authors}

65. Arditi C, Burnand B, Peytremann-Bridevaux, I. Additing non-randomised studies to a Cochrane review brings complementary information for healthcare stakeholders: an augmented systematic review and meta-analysis. BMC Health Services Research 2016;16:598.doi: 10.1186/s12913-016-1816-5.

66. Gidlow C, Johnston LH, Crone D, et al. A systematic review of the relationship between socio-economic position and physical activity. Health Educ J 2006;65:338- 